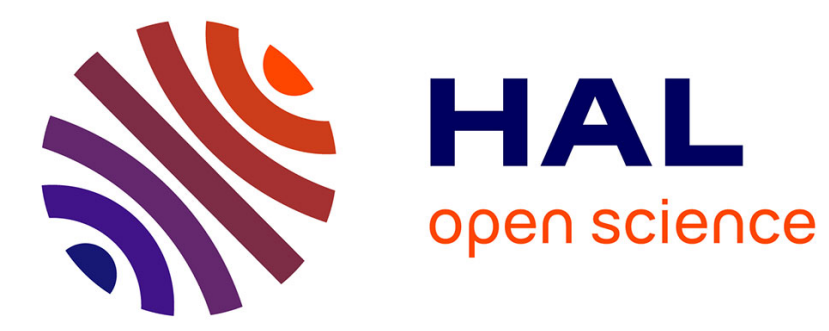

\title{
Morphological changes in early melanoma development: Influence of nutrients, growth inhibitors and cell-adhesion mechanisms
}

Clément Chatelain, Pasquale Ciarletta, Martine Ben Amar

\section{To cite this version:}

Clément Chatelain, Pasquale Ciarletta, Martine Ben Amar. Morphological changes in early melanoma development: Influence of nutrients, growth inhibitors and cell-adhesion mechanisms. Journal of Theoretical Biology, 2011, 290, pp.46. 10.1016/j.jtbi.2011.08.029 . hal-00743768

\section{HAL Id: hal-00743768 \\ https://hal.science/hal-00743768}

Submitted on 20 Oct 2012

HAL is a multi-disciplinary open access archive for the deposit and dissemination of scientific research documents, whether they are published or not. The documents may come from teaching and research institutions in France or abroad, or from public or private research centers.
L'archive ouverte pluridisciplinaire HAL, est destinée au dépôt et à la diffusion de documents scientifiques de niveau recherche, publiés ou non, émanant des établissements d'enseignement et de recherche français ou étrangers, des laboratoires publics ou privés. 


\section{Author's Accepted Manuscript}

Morphological changes in early melanoma development: Influence of nutrients, growth inhibitors and cell-adhesion mechanisms

Clément Chatelain, Pasquale Ciarletta, Martine Ben Amar

PII: S0022-5193(11)00432-2

DOI: doi:10.1016/j.jtbi.2011.08.029

Reference: YJTBI 6601

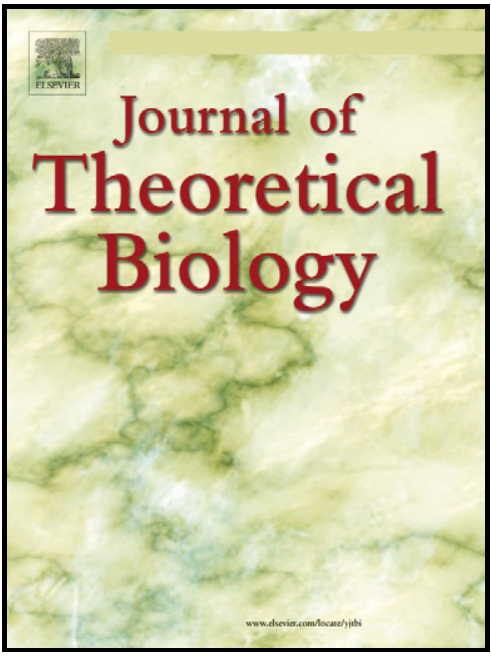

www.elsevier.com/locate/yjtbi

To appear in: $\quad$ Journal of Theoretical Biology

Received date: $\quad 15$ April 2011

Revised date: 22 August 2011

Accepted date: 23 August 2011

Cite this article as: Clément Chatelain, Pasquale Ciarletta and Martine Ben Amar, Morphological changes in early melanoma development: Influence of nutrients, growth inhibitors and cell-adhesion mechanisms, Journal of Theoretical Biology, doi:10.1016/j.jtbi.2011.08.029

This is a PDF file of an unedited manuscript that has been accepted for publication. As a service to our customers we are providing this early version of the manuscript. The manuscript will undergo copyediting, typesetting, and review of the resulting galley proof before it is published in its final citable form. Please note that during the production process errors may be discovered which could affect the content, and all legal disclaimers that apply to the journal pertain. 


\title{
Morphological changes in early melanoma development: influence of nutrients, growth inhibitors and cell-adhesion mechanisms
}

\author{
Clément Chatelain $^{\mathrm{a}}$, Pasquale Ciarletta ${ }^{\mathrm{b}}$, Martine Ben Amar ${ }^{\mathrm{a}, *}$ \\ ${ }^{a}$ Laboratoire de Physique Statistique, Ecole Normale Superieure, \\ UPMC Univ Paris 06, Université Paris Diderot, CNRS, \\ 24 rue Lhomond, 75005 Paris, France \\ ${ }^{b} \mathrm{CNRS}$ and Université Pierre et Marie Curie -Paris 06 \\ Institut Jean le Rond d'Alembert, UMR CNRS 7190, \\ 4 place Jussieu, case 162, 75005 Paris, France
}

\begin{abstract}
Current diagnostic methods for skin cancers are based on some morphological characteristics of the pigmented skin lesions, including the geometry of their contour. The aim of this article is to model the early growth of melanoma accounting for the biomechanical characteristics of the tumor micro-environment, and evaluating their influence on the tumor morphology and its evolution. The spatial distribution of tumor cells and diffusing molecules are explicitly described in a three-dimensional multiphase model, which incorporates general cell-to-cell mechanical interactions, a dependence of cell proliferation on contact inhibition, as well as a local diffusion of nutrients and inhibiting molecules. A two-dimensional model is derived in a lubrication limit accounting for the thin geometry of the epidermis. First, the dynamical and spatial properties of planar and circular tumor fronts are studied, with both numerical and analytical techniques. A WKB method is then developed in order to analyze the solution of the governing partial differential equations and to derive the threshold conditions for a contour instability of the growing tumor. A control parameter and a critical wavelength are identified, showing that high cell proliferation, high cell adhesion, large tumor radius and slow tumor growth correlate with the occurrence of a contour instability. Finally, comparing the theoretical results with a large amount of clinical data we show that our predictions describe accurately both the morphology of melanoma observed in vivo and its variations with the tumor growth rate. This study represents a fundamental step to understand more complex microstructural patterns observed during skin tumor growth. Its results have important implications for the improvement of the diagnostic methods for melanoma, possibly driving progress towards a personalized screening.
\end{abstract}

Keywords:

Contour instability, Tumor multiphase model, WKB analysis, Skin cancer morphology, Clinical dermatology 

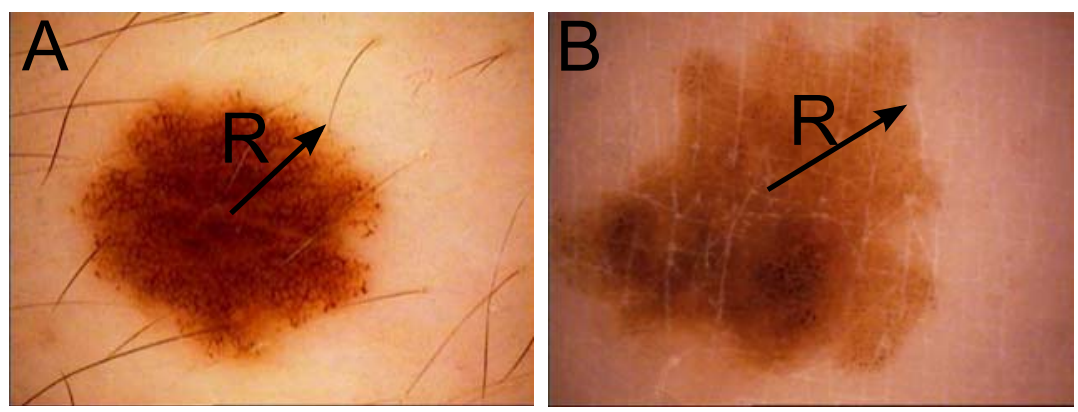

Figure 1: Dermatoscopy images illustrating the importance of morphological features in the clinical diagnosis of melanoma. Benign melanocytic nevus with a regular contour (A) and malignant melanoma with irregular contour and pigmentation (B). Courtesy of Dr. Harald Kittler (Department for Dermatology, University of Vienna) and Dr. Pascale Guitera (Sydney Melanoma Diagnostic Centre)

\section{Introduction}

Nowadays, skin cancer represents one third of all cancers that are diagnosed every year all over the world. It has been evaluated that over the past 31 years, more people have had skin cancer than all other cancers combined [1]. According to the American Cancer Society, more than two millions of new cases of non-melanoma skin cancers and about 70.000 of melanoma have been diagnosed in the United States in $2010^{1}$. Although melanoma represent less than five percent of all skin cancer, it is responsible for more than $75 \%$ of skin cancer death. While the incidence rate of many common cancer is falling, the one of melanoma increased by about $4 \%$ a year in the last 40 years, making its early diagnosis a priority for public health [2].

Melanoma arises usually from a preexisting benign tumor (nevi), but sometimes without clinical precursor lesion [3], and appears at the skin surface with an irregular pattern (Fig.1B). In the early stages, melanoma and nevi can present close clinical and histo-pathological properties and poor diagnosis is unfortunately common [4]. Indeed, extensive clinical investigations attempt to assess the malignancy of a lesion from the morphological changes of this mark at the skin surface $[5,6]$. In particular, dermoscopy images show that melanomas are usually characterized by an irregular growth pattern, and nevi by a regular circumferential growth [3]. Some morphological criteria to detect these lesions are worldwide adopted in clinical practice, such as the 'ABCDE rule' (Asymmetry, Border irregularity, Color variation, large Diameter, Evolution in time) or the '7-Point Checklist' (Atypical pigment network, Blue-white veil, Atypical vasculature, Irregular structures at the border, Irregular pigmentation, Dots or globules, White scar-like areas) [7]. After early detection and proper treatment, melanoma prognosis is excellent and the 10 -year survival rate is estimated to be about $95 \%$. Once it spreads, the prognosis becomes very poor despite recent advances in targeted therapy and immunotherapy $[8,9]$. According to the Cancer Research UK, for a stage III melanoma, which has spread to nearby lymph nodes, the survival rate drops to $15-24 \%$. However clinical examinations alone have been shown to have

\footnotetext{
${ }^{*}$ Corresponding author

Email address: benamar@lps.ens.fr (Martine Ben Amar)

${ }^{1}$ http://cancer.org/acs/groups/content/@nho/documents/skincancerpdf.pdf
} 
a rather low specificity and sensitivity in the early stage of melanoma evolution [10], and only $80 \%$ of melanoma are diagnosed before they spread [3].

Most melanomas are characterized by a first radial growth phase within the epidermis. The skin can be divided in three main layers: an inner layer, the hypodermis, constituted mainly of fibroblasts and adipocytes (this layer contains $50 \%$ of body fat insuring thermal insulation); an intermediate layer, the dermis, containing blood and lymphatic vasculature, hair follicles, glands, nerves and a network of collagen and elastic fibers providing skin elasticity; an external layer, the epidermis, which is an avascular stratified epithelium separated from the dermis by a basement membrane, a $50 \mathrm{~nm}$ thick wavy structure made of a dense extracellular matrix. The epidermis is mainly composed by keratinocytes that proliferate on the basement membrane and migrate to the skin surface while differentiating over a life-period of about 26 days, producing ultimately an insulating layer of lipids, keratin and dead keratinocytes called stratum corneum. Melanocytes are another important epidermis constituent, located on the basement membrane and producing melanin, responsible for skin pigmentation and protection from sun damage. In a normal skin, epidermal-melanin units are composed of an approximately constant ratio of 36 keratinocytes for one melanocyte [11].

Cutaneous melanoma results from a deregulation and anomalous proliferation of these pigmented cells. Melanomagenesis is a multistep process involving the modification of several molecular mechanisms (Fig.2): downregulation of proteins important for keratinocytemelanocyte communication and adhesion (E-cadherin, desmoglein and connexins), upregulation of proteins for melanocyte-melanocyte communication and adhesion (N-cadherin, Mel-CAM, integrin, ALCAM and connexins) [12], modification of signaling pathways controlling cell cycle and proliferation (eg. contact inhibition, complexes paracrines regulations) and immortalization by inactivation of tumor suppressor proteins [13]. Deviation from homeostasis leads in particular to an increase of the normally constant population ratio between melanocytes and keratinocytes, causing invasion of the surrounding tissue.

The aim of this article is to identify the parameters in the tumor micro-environment which might control some aspects of melanoma morphology and evolution, typical of aggressive neoplasia [15]. Eikenburry et al. [16] recently performed a numerical analysis concerning the interaction between melanoma and immune system, using a mathematical model to investigate the metastasis dynamics after primary tumor removal. In this work, we deal with the early radial growth of melanoma for its diagnostic importance, and we focus on the occurrence of shape irregularities at the border of the growing lesion. Contour instabilities have been studied in simplified single phase tumor models $[17,18]$ but there still lacks a description in a realistic framework that would take into account many important constituents of a complex tissue like the skin. In a recent review, Lowengrub et al. [19] provided an overview of the various approaches in tumor modeling, from discrete models describing individual cells [20] to continuum models at the tissue scale [21] and hybrid multiscale continuum-discrete models [22]. In particular, in the last ten years a wide range of problems related to tumor development has been successfully described in the context of mixture theory, as reviewed in $[19,23]$. These models are rather realistic but hard to handle analytically, being mostly used through numerical investigations $[24,25,26]$. Some of the numerical simulations actually exhibit growth instabilities, in various contexts and geometries [27], but little has been done to understand their physical and mathematical origins $[17,19,28]$. In a recent work [28] we have shown analytically and numerically the occurence of a contour instability in two-dimensional mixture models of tumour growth with nutrient limited proliferation. In the following, we develop a multiphase model for the early radial growth of melanoma in the epidermis, accounting for the complex structure of this skin 


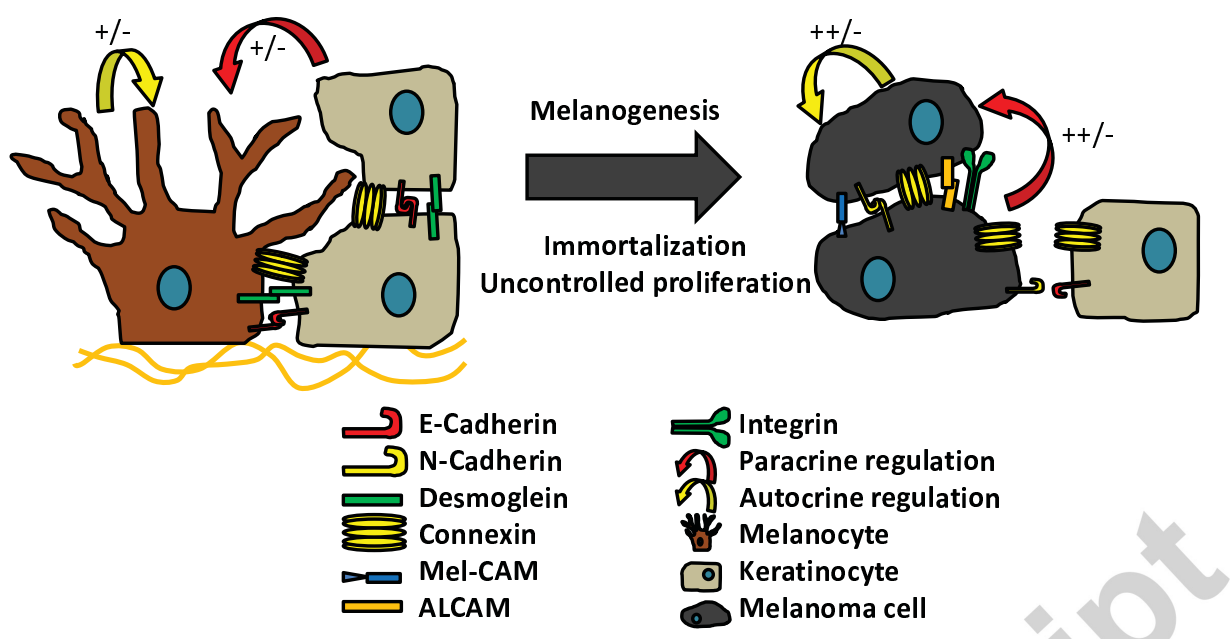

Figure 2: Interactions between keratinocytes, melanocytes and melanoma cells. Melanocytes and keratinocytes adhere via E-cadherin, desmogleins and connexins (gap junctions) which enable cell contact communication. Paracrine (resp. endocrine) regulation control normal melanocyte proliferation with the diffusion of cytokines and growth factors produced by the keratinocytes (resp. by the melanocyte itself). During melanogenesis, melanoma cells become immortal (inactivation of tumor suppressor proteins, such as p16), melanocyte-keratinocyte interaction proteins are down regulated (E-cadherin, desmoglein, connexins) and new proteins enable melanocyte-melanocyte interaction (N-cadherin, Mel-CAM, intergin, ALCAM and connexins). Melanoma cells become insensible to the inhibitor effect of some growth factors and produce new growth factors stimulating proliferation. For a detailed description of the different cytokines and growth factors see the review of Làzàr-Molnàr et al. [14].

layer and for the variety of mechanisms involved in melanoma development, but simple enough to have some physical insight into the morphogenesis of the lesion. We give a detailed stability analysis of this model using the method described in [28] with a lubrication approximation in the three-dimensional governing equations and investigating the effects of growth inhibitors and cell-adhesion regulation mechanisms.

The work is organized as follows. In Section 2 we present a three-dimensional (3D) model for early melanoma growth describing a mixture of a tumor cell phase and of an interstitial fluid. Taking into account the thin geometry of the epidermis, we derive from the 3D model a simplified two-dimensional (2D) model using the lubrication limit. In Section 3.1, we report the spatial and dynamical growth properties of a planar front. We focus in particular on three different regulation mechanisms for tumor cell proliferation: contact inhibition, autocrine inhibition and nutrient limited growth. In Section 3.1.2, we perform a linear stability analysis on the planar front, focusing on the occurrence of a wavy tumor contour with a typical finite wavelength. In Section 3.2.1, we extend our stability analysis for the growth of a circular tumor front, investigating the effects of a finite curvature on the morphology of the tumor lesion. Finally, we compare our theoretical predictions to the clinical observations of melanoma morphology and evolution rate, discussing the extent of our results for mathematical modeling and its applications in medical oncology. 


\section{Multiphase modeling of a melanoma spreading in the epidermis}

In this section we will define a multiphase mixture theory for modeling the early dynamics of a tumor lesion in the epidermis. First, we introduce the general constitutive equation for a twophase model. Secondly, we make the assumption of thin geometry and we derive the simplified governing equations for an averaged $2 \mathrm{D}$ model.

\subsection{Constitutive equations for a two-phase mixture}
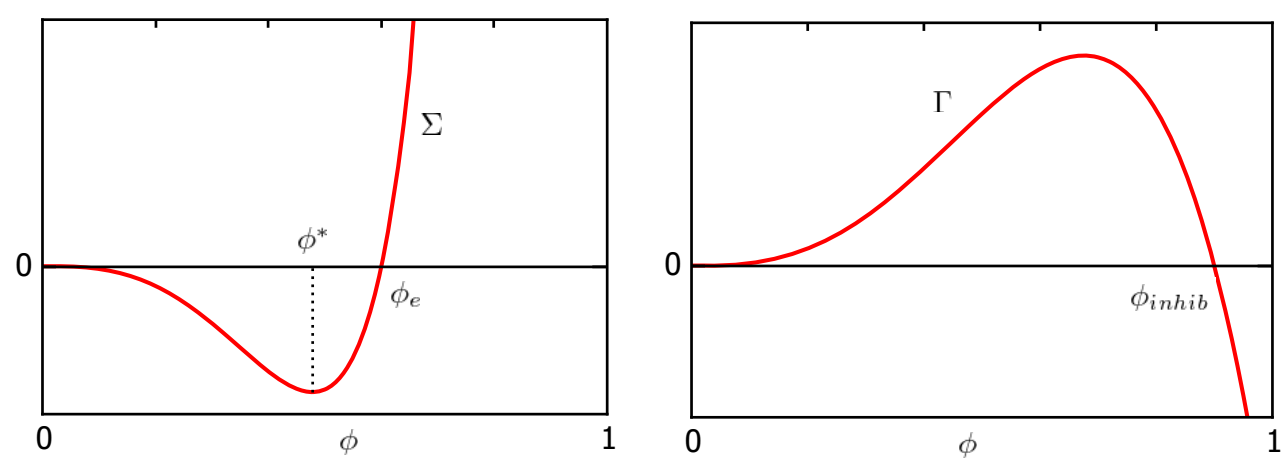

Figure 3: (A) Sketch of a mechanically consistent cell-to-cell potential interaction, attractive under a volume fraction $\phi_{e}$ and repulsive above. The presence of a minimum of interaction for $\phi=\phi *$ can eventually lead to a spinodal decomposition. (B) Sketch of a biologically relevant variation of the cell proliferation rate with cell volume fraction. At $\phi=\phi_{\text {inhib }}$ the apoptosis and necrosis compensate the cell divisions, and the cells are in a quiescent state.

Let us consider two main constituents of the epidermis: an interstitial fluid phase with volume fraction $\phi_{l}$ and velocity $\boldsymbol{v}_{l}$, containing soluble factors such as cell wastes and nutrients, and a proliferating cancerous cell phase with volume fraction $\phi_{c}$ and velocity $\boldsymbol{v}_{c}$. Keratinocytes are assumed to be dispersed in the interstitial fluid, the melanoma being a compact mass of tumor cells. Typical volume fractions in healthy tissues have been measured in rat skin at about $\phi_{c}=0.57-0.58$ [29] and in human epidermis at $\phi_{c}=0.63-0.87[30,31]$. Although simplificative (other cellular components of the epidermis are not considered), a two-phase model has the advantage to allow a deeper analytical treatment of the problem and, therefore, a better insight into the tumor dynamics and morphogenesis. The proliferation rate $\Gamma_{c}\left(\phi_{c}, n_{O_{2}}, n_{\text {inhib }}\right)$ of the cancerous cells depends on the tumor micro-environnement $[32,33]$ through modified endocrine/paracrine and cell cycle regulations. We will focus here on the dependence on the local concentrations $n_{\mathrm{O}_{2}}$ and $n_{\text {inhib }}$ of oxygen $[34,35,36]$ and of a generic growth inhibitor (eg. toxic cell wastes, cytokines) $[14,37,38]$, respectively, as well as on the influence of cell crowding [35, 39, 40, 41]. In particular Creasey et al. [39] reported the doubling time for human primary melanoma grown in culture, leading to a typical proliferation rate at $\Gamma_{c} \sim \gamma=0.2-0.67 \mathrm{day}^{-1}$. The mass balance equations can be written as follows:

$$
\rho_{i}\left(\frac{\partial \phi_{i}}{\partial t}+\nabla \cdot\left(\phi_{i} \boldsymbol{v}_{i}\right)\right) \underset{5}{=\rho_{i} \Gamma_{i}} \quad \text { with } i=l, c
$$


where the mass densities $\rho_{i}$ will be assumed constant and equal to the water mass density $\rho$, and we assume saturation of the mixture, imposing $\phi_{l}+\phi_{c}=1$ and $\Gamma_{l}=-\Gamma_{c}$ so that the incompressibility condition reads:

$$
\boldsymbol{\nabla} \cdot\left(\phi_{c} \boldsymbol{v}_{c}+\phi_{l} \boldsymbol{v}_{l}\right)=0
$$

For biological consistency, $\Gamma_{c}$ has to vanish, at given $n_{O_{2}}$ and $n_{i n h i b}$, both for $\phi_{c}=0$ and for a given $\phi_{\text {inhib }}>0$ corresponding to a balance between cell proliferation and contact inhibition $\left(\Gamma<0\right.$ for $\phi_{c}>\phi_{\text {inhib }}$ see Fig.3B).

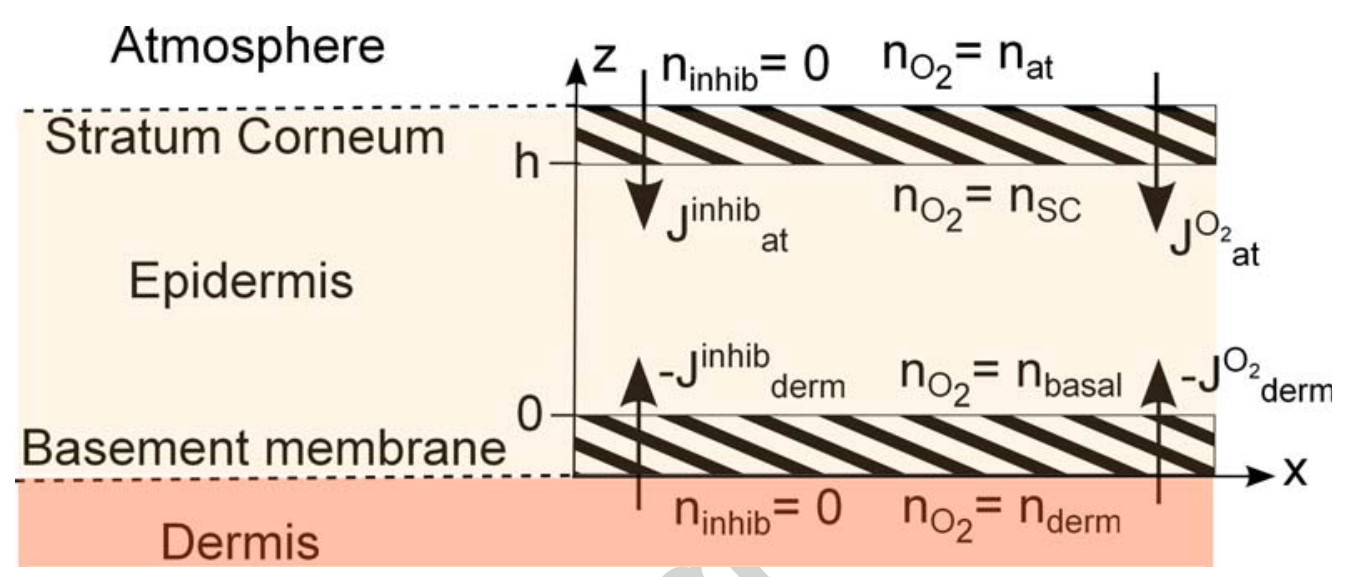

Figure 4: Epidermis layer with typical thickness $h \sim 0.1-1 \mathrm{~mm}[42,43]$ confined between an atmosphere/epidermis boundary at $z=h$ (Stratum Corneum) and an epidermis/dermis boundary at $z=0$ (Basement membrane). Oxygen and inhibitor have a fixed concentration in the atmosphere and in the dermis and diffuse through the epidermis boundaries generating fluxes $J_{a t}$ and $J_{d e r m}$ which depend on the local concentrations in $z=h\left(n_{S C}\right)$ and $z=0\left(n_{\text {basal }}\right)$.

Oxygen is consumed by cells at a rate $\delta_{O_{2}} \sim 1190-3030$ day $^{-1}[44,45]$ and diffuses in the interstitial fluid phase from the dermis and the atmosphere [44]. The oxygen fluxes $J_{a t}^{O_{2}}$ and $J_{\text {derm }}^{O_{2}}$ through the atmosphere/epidermis boundary (stratum corneum (SC)) and through the epidermis/dermis boundary (basement membrane) are given by the difference of concentration between the two sides of these boundaries (see Fig.4), as follows:

$$
\begin{aligned}
& J_{a t}^{O_{2}}=\alpha_{a t}^{O_{2}}\left(n_{a t}-n_{S C}\right) \\
& J_{\text {derm }}^{O_{2}}=\alpha_{\text {derm }}^{O_{2}}\left(n_{\text {basal }}-n_{\text {derm }}\right)
\end{aligned}
$$

with $\alpha_{a t}^{O_{2}}$ and $\alpha_{d e r m}^{O_{2}}$ being the permeability coefficients of the stratum corneum and of the basement membrane, respectively. In particular, Stücker et al. [44] measured the variation with depth of oxygen partial pressure in healthy skin, decreasing from $n_{S C}=78 \mathrm{mmHg}$ to $n_{\text {basal }}=25 \mathrm{mmHg}$ in the epidermis layer. According to Bedogni et al. [46], the skin micro-environment is mildly hypoxic at such concentrations, suggesting that a strong hypoxia can occur in the tumor center if tissue homeostasis is disrupted. Similarly, inhibitors of cell proliferation, such as toxic wastes and cytokines, are produced by tumor cells at a rate $\delta_{i n h i b}$, also diffusing through the atmosphere 
and the dermis. Assuming constant uptake/production rates, the advection-reaction-diffusion equations read:

$$
\begin{aligned}
& \frac{\partial n_{O_{2}}}{\partial t}+\boldsymbol{\nabla} \cdot\left(n_{O_{2}} \boldsymbol{v}_{l}\right)=\boldsymbol{\nabla} \cdot\left(\boldsymbol{D}_{O_{2}} \boldsymbol{\nabla} n_{O_{2}}\right)-\delta_{O_{2}} n_{O_{2}} \phi_{c} \\
& \frac{\partial n_{\text {inhib }}}{\partial t}+\boldsymbol{\nabla} \cdot\left(n_{\text {inhib }} \boldsymbol{v}_{l}\right)=\boldsymbol{\nabla} \cdot\left(\boldsymbol{D}_{\text {inhib }} \boldsymbol{\nabla} n_{\text {inhib }}\right)+\delta_{\text {inhib }} \phi_{c}
\end{aligned}
$$

where the parallel mobility coefficients $\left(D_{\mathrm{O}_{2}}^{/ /}\right.$and $\left.D_{\text {inhib }}^{/ /}\right)$can be different from the perpendicular coefficients $\left(D_{\mathrm{O}_{2}}^{z}\right.$ and $D_{\text {inhib }}^{z}$ ), mimicking an anisotropic diffusion. However, no experimental evidence exists that a longitudinal gradient of nutrients can provoke a transverse flux (or viceversa) so that a diagonal diffusion tensor is assumed. Johnson et al. [47] evaluated from experimental data that the parallel oxygen diffusion coefficient in the stratum corneum (external layer of skin composed of dead cells and rich in lipids) is about $D_{O_{2}}^{/ /}=39.7 \mathrm{~mm}^{2}$ day ${ }^{-1}$, while a slightly smaller perpendicular coefficient for oxygen diffusion is given as $D_{\mathrm{O}_{2}}^{z}=18.5-26.6 \mathrm{~mm}^{2}$ day $^{-1}$ using the distribution of oxygen partial pressure in the skin, as reported by Stücker et al [44]. The diffusion coefficients for the inhibitors are expected to be much smaller, because of their larger molecular volume. Considering the time scales involved in this growth process [48], the molecular concentrations can be assumed to be at diffusion equilibrium and we can neglect the left hand side of Eqs. $(5,6)$.

During malignant progression, melanoma cells acquire new cell-cell communication abilities, as reviewed by Hass et al. [12]. In particular, melanoma cells change interaction properties activating new adhesion molecules, e.g. N-cadherin, Mel-CAM ligand, $\alpha v \beta 3$ integrin, ALCAM and connexins. These interaction mechanisms, as well as other weakly non-local interactions between cells of the same species (eg. chemotaxis, haptotaxis), can be introduced in the expression of the Helmholtz free energy for the cell phase [49], which reads:

$$
F=\int_{\Omega} \psi\left(\phi_{c}\right)+\epsilon^{2} / 2\left|\nabla \phi_{c}\right|^{2} d \Omega
$$

with $\psi$ the free energy per unit of volume and $\epsilon \ll 1$. The main source of energy dissipation in the system can be assumed to be the viscous interaction between cells and the viscous drag of the cells moving relatively to the interstitial fluid. The energy dissipation can therefore be written

$$
W=\int_{\Omega} \frac{\tau \phi_{c}}{2}\left(\boldsymbol{v}_{c}-\boldsymbol{v}_{l}\right)^{2}+\frac{\mu \phi_{c}}{2}\left(\boldsymbol{\nabla} \cdot \boldsymbol{v}_{c}\right)^{2} d \Omega
$$

with $\mu$ the cell phase viscosity and $\tau$ a constant friction parameter. Measurements of the Darcy law in rat skin $[31,50]$ gives an estimate of this friction parameter in the range $\tau=7.24-$ $87.0 \mathrm{~mm}^{-2} \mathrm{mmHg}$ day. Following Doi and Onuki [51] we use Rayleigh's variational principle indicating that the overdamped system dynamics can be obtained by minimizing the Rayleighian $\mathcal{R}=W+d F / d t$ with respect to the velocities $\boldsymbol{v}_{c}$ and $\boldsymbol{v}_{l}$. The incompressibility constraint given in Eq.(2) is accounted for by adding a Lagrange multiplier $p$ so, using Eq.(1) the Rayleighian reads:

$$
\mathcal{R}=\int_{\Omega}[\underbrace{\frac{\tau \phi_{c}}{2}\left(\boldsymbol{v}_{c}-\boldsymbol{v}_{l}\right)^{2}+\frac{\mu}{2} \phi_{c}\left(\boldsymbol{\nabla} \cdot \boldsymbol{v}_{c}\right)^{2}}_{\text {dissipation }}+\underbrace{\left(\frac{\partial \psi}{\partial \phi_{c}}-\epsilon^{2} \Delta \phi_{c}\right)\left(-\boldsymbol{\nabla} \cdot\left(\phi_{c} \boldsymbol{v}_{c}\right)+\Gamma_{c}\right)}_{\text {variation of free energy }}-\underbrace{p \boldsymbol{\nabla} \cdot\left(\phi_{c} \boldsymbol{v}_{c}+\phi_{l} \boldsymbol{v}_{l}\right)}_{\text {incompressibility }}] d \Omega
$$


Minimizing this functional with respect to $\boldsymbol{v}_{c}$ and $\boldsymbol{v}_{l}$ gives the governing equilibrium equations for the phases:

$$
\begin{aligned}
& \tau \phi_{c}\left(\boldsymbol{v}_{c}-\boldsymbol{v}_{l}\right)+\phi_{c} \boldsymbol{\nabla}\left(\frac{\partial \psi}{\partial \phi_{c}}-\epsilon^{2} \Delta \phi_{c}\right)+\phi_{c} \boldsymbol{\nabla} p-\mu \phi_{c} \Delta \boldsymbol{v}_{c}=0 \\
& \tau \phi_{c}\left(\boldsymbol{v}_{l}-\boldsymbol{v}_{c}\right)+\phi_{l} \boldsymbol{\nabla} p=0
\end{aligned}
$$

These equations can be interpreted as the mechanical equilibrium in each phase for a system with negligible inertia, with $p$ the hydrostatic pressure and

$$
\frac{\partial \psi}{\partial \phi_{c}}-\epsilon^{2} \Delta \phi_{c}=\Sigma
$$

$\Sigma$ being the excess pressure generated by cell-cell interactions. A typical order of magnitude $\chi$ for this pressure $\Sigma$ can be estimated from the interstitial fluid pressures reported in the review by Jain [30] for various tumors and tissues: a value $\chi \sim 1 \mathrm{mmHg}$ is found in healthy rat skin, while $\chi \sim 10 \mathrm{mmHg}$ for carcinoma in rat skin. This is compatible with the results both of Wiig et al. [29] (giving $\chi \sim 1 \mathrm{mmHg}$ for rat skin) and of Kristensen et al. [52] (reporting $\chi=1.6-28.5 \mathrm{mmHg}$ for human melanoma). For physical and biological consistency, $\Sigma\left(\phi_{c}, \Delta \phi_{c}\right)$ has to vanish for $\phi_{c} \rightarrow 0$ and to diverge for $\phi_{c} \rightarrow 1$ showing a strong repulsion at high cancerous cell concentration, as depicted in Fig.3A. The presence of a minimum in $\Sigma$ for a given volume fraction $\phi *$ can eventually lead to a spinodal decomposition due to negative diffusion coefficients for $\phi_{c}<\phi *$. However the development of these structures is obtained for specific parameters of the model, as we will show in a forthcoming work. We will consider here only situations where this instability doesn't appear and we can therefore neglect the dependence of $\Sigma$ on the derivatives of $\phi_{c}(\epsilon=0)$. Eliminating $p$ from Eqs. $(10,11)$ and recalling the saturation constraint $\phi_{c}+\phi_{l}=1$ we get the relation for the relative velocity between the cell and fluid phases

$$
\boldsymbol{v}_{c}-\boldsymbol{v}_{l}=\left(\left(1-\phi_{c}\right) \phi_{c} / \tau\right) \boldsymbol{\nabla} \cdot\left(-\Sigma \mathbf{1}+\boldsymbol{\nabla} \boldsymbol{v}_{c}\right)
$$

\subsection{Lubrication approximation for thin geometry}

During its radial phase, the melanoma spreads superficially in the epidermis layer. The thickness $h$ of the epidermis can be considered much smaller than the radial size $L$ of the tumor (typically $h=0.1-1 \mathrm{~mm}$ and $L \sim 5 \mathrm{~mm}[42,43,6]$ ), and a $2 \mathrm{D}$ approximation in thin geometry can be formulated, using the scalings $x=\hat{x} L, y=\hat{y} L, z=\hat{z} h, v_{i, x, y}=\hat{v}_{i, x, y} V_{/ /}, v_{i, z}=\hat{v}_{i, z} V_{z}$ with $i=(c, l)$, and $\Sigma=\chi \hat{\Sigma}$, with $h / L \ll 1$ and $V_{z} / V_{/ /} \ll 1$. At the leading order in $h / L$ and $V_{z} / V_{/ /}$, Eq.(13) can be rewritten as:

$$
\begin{aligned}
& \hat{v}_{c, x}-\hat{v}_{l, x}=\frac{\left(1-\phi_{c}\right) \phi_{c} \chi}{\tau L V_{/ /}} \frac{\partial \hat{\Sigma}}{\partial \hat{x}}+\frac{\left(1-\phi_{c}\right) \phi_{c} \mu}{\tau h^{2}} \frac{\partial^{2} \hat{v}_{c, x}}{\partial \hat{z}^{2}} \\
& \hat{v}_{c, y}-\hat{v}_{l, y}=\underbrace{\frac{\left(1-\phi_{c}\right) \phi_{c \chi}}{\tau L V_{/ /}}}_{\theta_{1}} \frac{\partial \hat{\Sigma}}{\partial \hat{y}}+\underbrace{\frac{\left(1-\phi_{c}\right) \phi_{c} \mu}{\tau h^{2}}}_{\theta_{2}} \frac{\partial^{2} \hat{v}_{c, y}}{\partial \hat{z}^{2}} \\
& \hat{v}_{c, z}-\hat{v}_{l, z}=\underbrace{\frac{\left(1-\phi_{c}\right) \phi_{c \chi}}{\tau h V_{z}}}_{\theta_{3}} \frac{\partial \hat{\Sigma}}{\partial \hat{z}}+\underbrace{\frac{\left(1-\phi_{c}\right) \phi_{c} \mu}{\tau h^{2}}}_{\theta_{4}} \frac{\partial^{2} \hat{v}_{c, z}}{\partial \hat{z}^{2}}
\end{aligned}
$$


From biological data $[29,31,50,52,53]$, we estimate $\theta_{3} \sim 33.3-1.43 \times 10^{4} \gg 1$ and we can therefore neglect the left hand side in Eq.(16) for the $z$ component. Comparing the viscous term and the $\Sigma$ term, we get $\theta_{3} / \theta_{4}=3.83 \times 10^{5}-1.56 \times 10^{9} \gg 1$ [54], showing that Eq.(16) can be rewritten as:

$$
\frac{\partial \Sigma}{\partial z} \cong 0
$$

Recalling that $\Sigma=\Sigma\left(\phi_{c}\right)$, this equation implies in particular that $\partial \phi_{c} / \partial z=0$, i.e. a homogeneous tumor cell volume fraction along $z$. Comparing the viscous term and the $\Sigma$ term in the $x$ and $y$ components given by Eqs. $(14,15)$, we get that $\theta_{1} / \theta_{2}=3.46-4.1 \times 10^{5} \gg 1$. The horizontal velocity is therefore given by:

$$
\boldsymbol{v}_{c, / /}-\boldsymbol{v}_{l, / /}=\frac{\left(1-\phi_{c}\right) \phi_{c}}{\tau} \nabla_{/ /} \Sigma\left(\phi_{c}\right)
$$

where we use the notation $\boldsymbol{x}_{/ /}=\boldsymbol{x}-\left(\boldsymbol{x} \cdot \boldsymbol{e}_{z}\right) \boldsymbol{e}_{z}$ for describing a geometrical projection on a plane perpendicular to the unit vector $\boldsymbol{e}_{z}$.

Integrating Eq.(2) between $z=0$ and $z=h$ with the boundary condition $V_{m i x, z}(0)=V_{m i x, z}(h)=$ 0 and dividing by $h$ leads to the two dimensional incompressibility condition

$$
\boldsymbol{\nabla}_{/ /} \cdot\left\langle\boldsymbol{V}_{\text {mix }, / /}\right\rangle=0
$$

with $\boldsymbol{V}_{\text {mix }}=\phi_{c} \boldsymbol{v}_{c}+\phi_{l} \boldsymbol{v}_{l}$ the total mixture velocity and the average quantities defined by $\langle(\cdot)\rangle=$ $h^{-1} \int_{0}^{h}(\cdot) d z$. For a general mixture Eq.(19) is not sufficient to close the system of equations and one would need additional assumptions on the velocities [55]. However in the following we will consider systems with planar or radial symmetry, the unrealistic planar case being simpler for the analytical treatment. In these situations the symmetry restrictions provide the missing equations, and an explicit solution can be given with respect to the phase velocities, as follows:

$$
\begin{aligned}
& \left\langle\boldsymbol{v}_{c, / /}\right\rangle=-K\left(\phi_{c}\right) \nabla_{/ /} \Sigma\left(\phi_{c}\right) \\
& K\left(\phi_{c}\right)=\frac{\left(1-\phi_{c}\right)^{2} \phi_{c}}{\tau} \nabla_{/ /} \Sigma\left(\phi_{c}\right)
\end{aligned}
$$

which is an extension of the Darcy law for the two-phase mixture. We notice that for a system without particular symmetry an additional constitutive equation on the velocity fields must be given.

Integrating Eqs. $(1,5,6)$ between $z=0$ and $z=h$, and dividing by $h$, we can rewrite the governing equations in the following averaged form:

$$
\begin{aligned}
& \frac{\partial \phi_{c}}{\partial t}-\boldsymbol{\nabla}_{/ /} \cdot\left(\phi_{c} K\left(\phi_{c}\right) \boldsymbol{\nabla}_{/ / \Sigma}\right)=\tilde{\Gamma}_{c}\left(\phi_{c},\left\langle n_{O_{2}}\right\rangle,\left\langle n_{\text {inhib }}\right\rangle\right) \\
& 0=\boldsymbol{\nabla}_{/ /} \cdot\left(D_{O_{2}}^{/ /} \boldsymbol{\nabla}_{/ /}\left\langle n_{O_{2}}\right\rangle\right)-\delta_{O_{2}} \phi_{c}\left\langle n_{O_{2}}\right\rangle+\frac{D_{O_{2}}^{z}}{h}\left[\frac{\partial n_{O_{2}}}{\partial z}\right]_{0}^{h} \\
& 0=\boldsymbol{\nabla}_{/ /} \cdot\left(D_{\text {inhib }}^{/ /} \nabla_{/ /}\left\langle n_{\text {inhib }}\right\rangle\right)+\delta_{\text {inhib }} \phi_{c}+\frac{D_{\text {inhib }}^{z}}{h}\left[\frac{\partial n_{\text {inhib }}}{\partial z}\right]_{0}^{h}
\end{aligned}
$$

where $\tilde{\Gamma_{c}}=h^{-1} \int_{0}^{h} \Gamma_{c}\left(\phi_{c}, n_{O_{2}}, n_{\text {inhib }}\right) d z$. In order to determine the last term in Eqs. $(23,24)$, let us look for a solution to Eq.(5) mainly two dimensional but with a tiny contribution that has a sharp variation with the thickness $h$ in the form:

$$
n_{O_{2}}(x, y, z)=n_{0}(x, y)+(h / L)^{2} n_{1}\left(x, y, z(L / h)^{2}\right)+(h / L)^{4} n_{2}\left(x, y, z(L / h)^{2}\right)+o\left((h / L)^{4}\right)
$$


Setting $n_{S C}=n_{O_{2}}(z=h)$ and $n_{\text {basal }}=n_{O_{2}}(z=0)$ the boundary conditions given by Eqs. $(3,4)$ can be rewritten at the leading order in $h / L$ as follows:

$$
\begin{aligned}
& D_{O_{2}}^{z} \frac{\partial n_{O_{2}}}{\partial z}(h)=D_{O_{2}}^{z} \frac{\partial n_{1}}{\partial z(L / h)^{2}}(h)=J_{a t}^{O_{2}}=\alpha_{a t}^{n}\left(n_{a t}-n_{0}\right)+O\left((h / L)^{2}\right) \\
& D_{O_{2}}^{z} \frac{\partial n_{O_{2}}}{\partial z}(0)=D_{O_{2}}^{z} \frac{\partial n_{1}}{\partial z(L / h)^{2}}(0)=J_{\text {derm }}^{O_{2}}=\alpha_{\text {derm }}^{n}\left(n_{0}-n_{\text {derm }}\right)+O\left((h / L)^{2}\right)
\end{aligned}
$$

Finally, the equation for the mean concentration $N=\left\langle n_{O_{2}}\right\rangle$ reads:

$$
\boldsymbol{\nabla}_{/ /} \cdot\left(D_{O_{2}}^{/ /} \boldsymbol{\nabla}_{/ /} N\right)-\delta_{n} \phi_{c} N+D_{b, N}\left(N_{e}-N\right)=0
$$

with $D_{b, N}=1 /\left(h\left(\alpha_{a t}^{O_{2}}+\alpha_{d e r m}^{O_{2}}\right)\right)$ and $N_{e}=\left(\alpha_{a t}^{O_{2}} n_{a t}+\alpha_{d e r m}^{O_{2}} n_{d e r m}\right) /\left(\alpha_{a t}^{O_{2}}+\alpha_{d e r m}^{O_{2}}\right)$. A similar analysis for the mean inhibitor concentration $I=\left\langle n_{\text {inhib }}\right\rangle$ gives:

$$
\boldsymbol{\nabla}_{/ /} \cdot\left(D_{\text {inhib }}^{/ /} \boldsymbol{\nabla}_{/ /} I\right)+\delta_{\text {inhib }} \phi_{c}-D_{b, I} I=0
$$

with $D_{b, I}=1 /\left(h\left(\alpha_{a t}^{i n h i b}+\alpha_{d e r m}^{i n h i b}\right)\right)$. Notice that the neglected terms in Eqs.(28,29) are of order $h / L$ while these equations include terms of order $L / h$ and 1 . In particular, $D_{b, N}, D_{b, I}$ diverge at vanishing $h$, imposing $N=N_{e}$ and $I=0$, and the resulting system is governed by the following equation:

$$
\frac{\partial \phi_{c}}{\partial t}-\nabla_{/ /} \cdot\left(\phi_{c} K\left(\phi_{c}\right) \nabla_{/ / \Sigma}\right)=\tilde{\Gamma_{c}}\left(\phi_{c}\right)
$$

which is the mean equation for tumor cell mobility in the simplified 2D model, considering only contact inhibition of growth.

\subsection{Governing equations in dimensionless form}

The governing equations can be rewritten in terms of the dimensionless quantities $\bar{x}=$ $\sqrt{\tau \gamma / \chi} x, \bar{t}=\gamma t, \bar{I}=\left(\tau \gamma D_{\text {inhib }}^{/ /} /\left(\delta_{\text {inhib } \chi}\right)\right) I, \bar{N}=N_{e}^{-1} N, \bar{\Sigma}=\chi^{-1} \Sigma, \bar{K}\left(\phi_{c}\right)=\tau K\left(\phi_{c}\right), \bar{\Gamma}=(\gamma h)^{-1} \tilde{\Gamma}_{c}$, $\gamma$ being a characteristic value of the proliferation rate $\Gamma_{c}$. Dropping the bars, the tildes, the subscripts for the cell phase and the parallel indices in the following, we get the governing equations together with the boundary conditions at the tumor border $x_{b}$. In particular, assuming a stress free boundary, with unit normal vector $\boldsymbol{n}$ at $\boldsymbol{x}_{b}$, imposing $\phi\left(\boldsymbol{x}_{b}\right)=\phi_{e}$ with $\Sigma\left(\phi_{e}\right)=0$ [56], the dimensionless governing equations read:

$$
\begin{array}{rll}
\frac{\partial \phi}{\partial t}+\boldsymbol{\nabla} \cdot(\phi \boldsymbol{v})=\Gamma(\phi, N, I), & \phi\left(\boldsymbol{x}_{b}\right)=\phi_{e} \\
\boldsymbol{v}=-K(\phi) \boldsymbol{\nabla} \Sigma, & \boldsymbol{n} \cdot d \boldsymbol{x}_{b} / d t=\boldsymbol{n} \cdot \boldsymbol{v}\left(\boldsymbol{x}_{b}\right)=V_{b} \\
0=\Delta N-\beta_{1} \phi_{c} N+\beta_{2}(1-N), & N\left(\boldsymbol{x}_{b}\right)=1 \\
0=\Delta I+\phi_{c}-\alpha I, & I\left(\boldsymbol{x}_{b}\right)=0
\end{array}
$$

The governing parameters are the dimensionless functions $\Gamma$ and $\Sigma$, and the dimensionless constants $\alpha=D_{b, I} \chi /\left(\tau \gamma D_{\text {inhib }}^{/ /}\right), \beta_{1}=\chi \delta_{O_{2}} /\left(\tau \gamma D_{O_{2}}^{/ /}\right), \beta_{2}=\chi D_{b, N} /\left(\tau \gamma \delta_{O_{2}}\right)$. The choice of the time unit is motivated by giving the growth rate in terms to the typical cell proliferation rate $\gamma$, in order to validate the results with clinical data on melanomas [48]. 


\begin{tabular}{|l|l|c|}
\hline Parameter & Value & Ref \\
\hline Equilibrium cell volume fraction $\phi_{e}$ & $0.57-0.87$ & {$[29,31]$} \\
\hline Typical proliferation rate $\gamma$ & $0.2-0.67$ day $^{-1}$ & {$[35,39,57]$} \\
\hline Oxygen consumption rate $\delta_{O_{2}}$ & $1190-3030$ day $^{-1}$ & {$[44,45]$} \\
\hline $\begin{array}{l}\text { Parallel oxygen diffusion coefficient } \\
\text { in the epidermis } D_{O_{2}}^{/ /}\end{array}$ & $39.7 \mathrm{~mm}^{2}$ day $^{-1}$ & {$[47]$} \\
\hline $\begin{array}{l}\text { Perpendicular oxygen diffusion coefficient } \\
\text { in the epidermis } D_{\mathrm{O}_{2}}^{z}\end{array}$ & $18.5-26.6 \mathrm{~mm}^{2}$ day $^{-1}$ & {$[44]$} \\
\hline $\begin{array}{l}\text { Oxygen partial pressure in } \\
\text { the stratum corneum } n_{S C}\end{array}$ & $78 \mathrm{mmHg}$ & {$[44]$} \\
\hline $\begin{array}{l}\text { Oxygen partial pressure in } \\
\text { the basal layer } n_{\text {basal }}\end{array}$ & $25 \mathrm{mmHg}$ & {$[44]$} \\
\hline $\begin{array}{l}\text { Typical pressure resulting from } \\
\text { cell-cell interactions } \chi\end{array}$ & $1-28.5 \mathrm{mmHg}$ & {$[29,30,52]$} \\
\hline cell phase viscosity $\mu$ & $300-10^{3} \mathrm{~Pa} \mathrm{~s}$ & {$[54]$} \\
\hline Friction between cell and fluid phases $\tau$ & $7.24-87.0 \mathrm{~mm}^{-2} \mathrm{mmHg}$ day & {$[31,50]$} \\
\hline Epidermis thickness $H$ & $0.1-1 \mathrm{~mm}$ & {$[6,42,43]$} \\
\hline Typical cell death rate in the absence of nutrients $\delta$ & $0.05-1.65$ & {$[39,57]$} \\
\hline
\end{tabular}

Table 1: Physical parameters estimated from experimental studies in clinical oncology.

\section{Linear stability analysis for planar and radial tumor fronts}

We consider the horizontal spreading in the early development of melanoma, occurring before the vertical phase which concerns penetration inside the dermis. First, we assume a negligible mechanical resistance exerted by the underlying healthy tissue. Starting from an initial circular shape with radius $R_{0}$, here we study both analytically and numerically the evolution of the tumor mass, as given by Eqs. $(31,32,33,34)$.

In order to understand the influence of the different growth mechanisms driving melanoma evolution, we will consider three distinct regimes: a contact-inhibition limited growth $(\mathrm{CI})$, inhibitor-diffusion limited growth (ID) and oxygen-diffusion limited growth (OD). These regimes correspond to the values of material parameters reported in Table 2. For the sake of completeness, we remind that most of the results hold for general expressions $\Gamma$ and $\Sigma$ satisfying the constraints explained in Section (2.1). For the (OD) regime, typical cell death and mass decrease rate in the absence of nutrients can be estimated from the results of Creasey et al. [39] and Cardenas et al. [57], corresponding to $\delta=0.05-1.65$ for different cell lines.

\subsection{Contour instability of a planar tumor front}

Let us first consider the limit case of an infinite tumor radius $R$, discussing later the more realistic finite case. This limit corresponds to the one dimensional growth along a $x$ axis of a tumor initially invariant along $y\left(\phi(x, y, 0)=\phi_{i n i}(x)\right)$. Numerical simulations of the three regimes in such geometry give travelling wave solutions with a front at $x=L(t)$ moving at a constant velocity $L(t)=L_{0}+U t$, independently of the initial conditions of the initial conditions (see Fig.5), to be compared with the constant growth rate observed in vivo [48]. Using the moving 


\begin{tabular}{|c|c|c|c|c|}
\hline Regime & limit & $N$ & $I$ & $\Gamma$ \\
\hline Contact Inhibition (CI) & $\beta_{2} \rightarrow+\infty, \alpha \rightarrow+\infty$ & $=1$ & $=0$ & $\left(\phi_{\text {inhib }}-\phi\right) \phi^{3}$ \\
\hline Inhibitor Diffusion (ID) & $\alpha \rightarrow+\infty$ & $=1$ & & $(1-\delta I) \phi$ \\
\hline Oxygen Diffusion (OD) & $\beta_{2} \rightarrow+\infty$ & & $=0$ & $(N-\delta) \phi$ \\
\hline
\end{tabular}

Table 2: Three different regulation mechanisms for the tumor cell proliferation rate $\Gamma$, considered separately in three regimes: regulation by contact inhibition (through the tumor cell volume fraction $\phi$ ), regulation by the local concentration $I$ in a growth inhibitor produced by the cells or regulation by the local oxygen concentration $N$.

reference coordinate $z=x-L(t)$, a travelling wave solution $\left(\phi_{0}(z), N_{0}(z), I_{0}(z)\right)$ propagating from $z=-\infty$ satisfies the following ordinary differential equations:

$$
\begin{aligned}
& -U \phi_{0}^{\prime}-\left(\phi_{0} K\left(\phi_{0}\right) \Sigma_{\phi}\left(\phi_{0}\right) \phi_{0}^{\prime}\right)^{\prime}=\Gamma\left(\phi_{0}, N_{0}, I_{0}\right) \\
& 0=N_{0}^{\prime \prime}-\beta_{1} \phi_{0} N_{0}+\beta_{2}\left(1-N_{0}\right) \\
& 0=I_{0}^{\prime \prime}+\phi_{0}-\alpha I_{0}
\end{aligned}
$$

where the prime denotes the derivative with respect to $z$ and $\Sigma_{\phi}=d \Sigma / d \phi$.

\subsubsection{Properties of the travelling wave solutions}

In the (CI) regime and for $\phi_{\text {inhib }}>\phi_{e}$, a solution $\phi_{0}(z)$, found also numerically, is a decreasing function of $z$ (see Fig.5). An asymptotic analysis of Eq.(35) for $z \rightarrow-\infty$ shows that the cell volume fraction in the tumor center is $\Phi_{0}=\phi_{\text {inhib }}$, corresponding to a complete contact inhibition of growth $\left(\Gamma\left(\phi_{i n h i b}\right)=0\right)$. All the proliferating activity is thus confined in an outer ring of typical size $l_{p}$. Although a solution with $\phi=0$ in the tumor center is coherent with the asymptotic analysis, it cannot be observed because the uniform solution $\phi=0$ is unstable for small perturbations of $\phi$. If $\phi_{i n h i b}<\phi_{e}$ another solution is given by a decreasing function of $z$ with $U<0$, corresponding to a regressing tumor $[58,59,60]$.

In the (ID) regime, an asymptotic analysis in $z=-\infty$ of Eqs. $(35,37)$ shows that the cell volume fraction in the tumor center is $\Phi_{0}=\alpha / \delta$, corresponding to the ratio between the evacuation rate of inhibitors and the inhibition rate of proliferation. For $\Phi_{0}>\phi_{e}$ the solution is a decreasing function $\phi_{0}(z)$ with higher cell volume fraction at its center, and for $\Phi_{0}<\phi_{e}, \phi_{0}$ has a maximum near the tumor border at $z=z_{m}$, as depicted Fig.(5C,5D). As previously discussed, a solution with $\phi=0$ in the tumor center satisfy the asymptotic analysis but is not a stable solution. The distribution of inhibitors in the tumor can be easily determined from Eq.(37), being:

$$
I_{0}(z)=\frac{1}{\sqrt{\alpha}} \int_{z}^{0} \phi_{0} \sinh \left(\sqrt{\alpha}\left(z-z^{\prime}\right)\right) d z^{\prime}-\frac{\Phi_{0}}{\alpha} \sinh (\sqrt{\alpha} z)
$$

The size of the proliferating region $l_{p}$ is then given by the typical size of decay of $I$ near the tumor border, with a scaling given by:

$$
l_{p} \sim 1 / \sqrt{\alpha}
$$

Finally, in the (OD) regime the asymptotic analysis of Eqs. $(35,36)$ gives a nutrient concentration $\delta$ and a cell volume fraction $\left(\beta_{2} / \beta_{1}\right)\left(\delta^{-1}-1\right)=\Phi_{0}$ in the tumor center (for $\left.z \rightarrow-\infty\right)$. Again, if $\Phi_{0}>\phi_{e}$ the solution has a maximum in cell volume fraction near the tumor border at $z=z_{m}$ and is monotonic otherwise, as depicted Fig.(5E,5F). Looking for solutions to the homogeneous 
Necrotic core
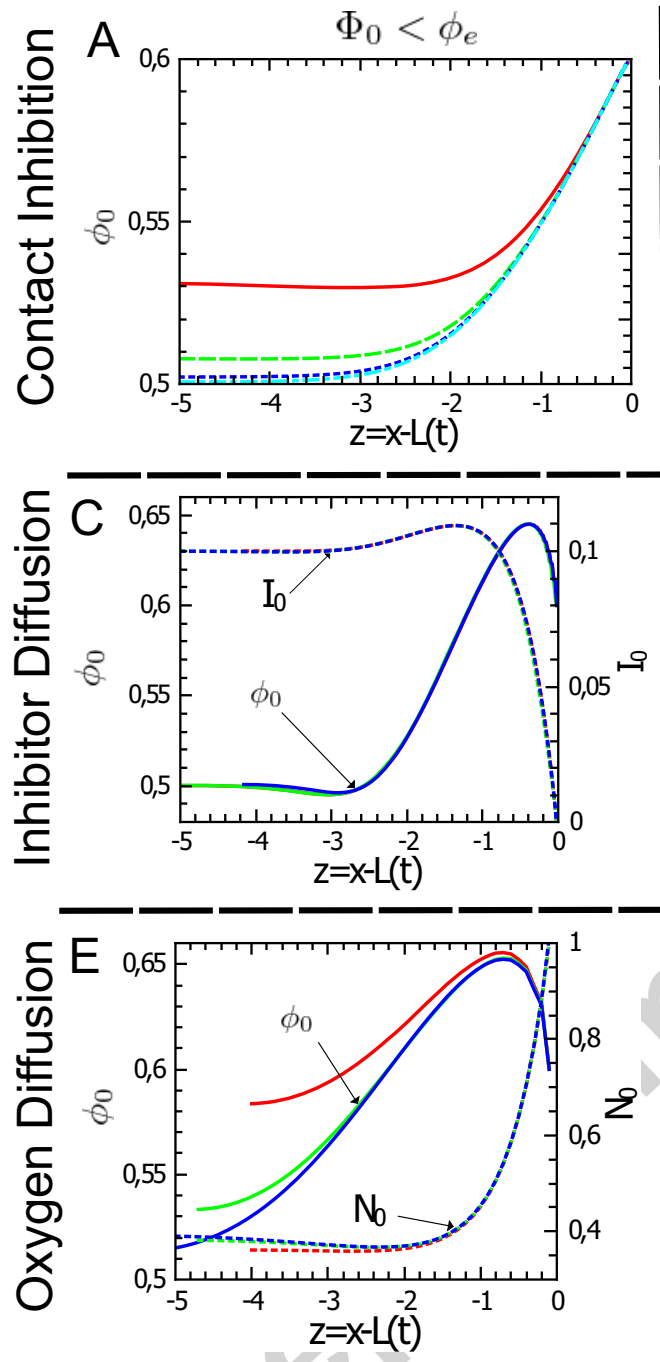

Quiescent core
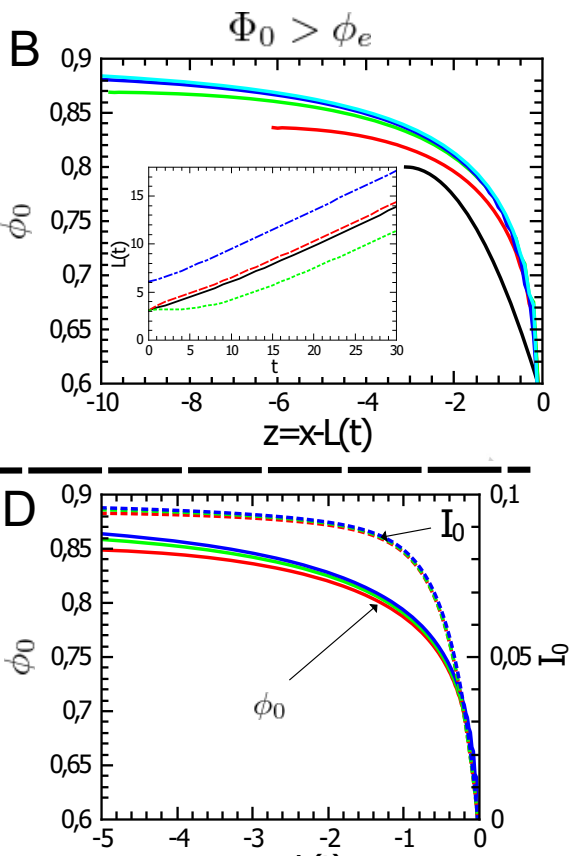

$\mathrm{z}=\mathrm{x}-\mathrm{L}(\mathrm{t})$

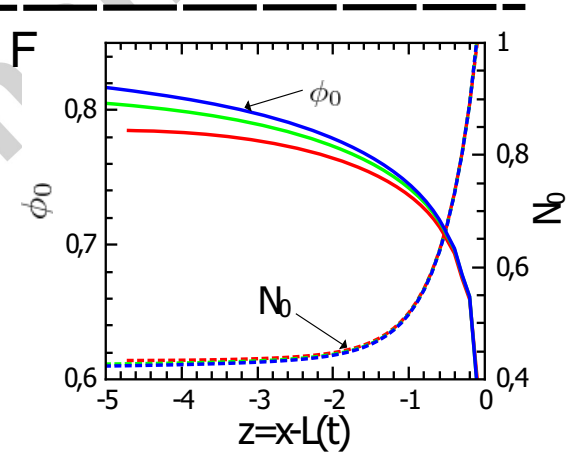

Figure 5: (A,B) $\phi_{0}$ from 1D numerical simulation of the model in the (CI) regime at time $t=0$ (black), 10 (red), 20 (green), 30 (blue), 40 (blue). The simulation parameters are $\phi_{e}=0.6, \phi_{\text {inhib }}=0.5$ (A) and $\phi_{\text {inhib }}=0.9$ (B), and the initial condition is $\phi_{\text {ini }}(x)=\left(\phi_{a}-\left(\phi_{a}-\phi_{e}\right)\left(x / R_{0}\right)^{2}\right)$ with $\phi_{a}=0.8$ and $R=3$. In the inset, the linear growth of $L$ after a transitory regime, independent of the initial conditions, here $R=3$ and $\phi_{a}=0.3$ (green dashed line), 0.8 (black continuous line), 0.95 (red dashed line), and $R=6$ and $\phi_{a}=0.8$ (blue dashed line). (C,D) $\phi_{0}$ (continuous lines) and $I_{0}$ (dashed lines) from 1D numerical simulation of the model in the (ID) regime at time $t=5$ (red), 10 (green), 15 (blue). $\phi_{e}=0.6, \delta=10$, $\alpha=5$ (C) and $\alpha=9$ (D). (E,F) $\phi_{0}$ (continuous lines) and $N_{0}$ (dashed lines) from 1D numerical simulation of the model in the (OD) regime at time $t=5$ (red), 10 (green), 15(blue). $\phi_{e}=0.6, \delta=0.4, \beta_{1}=4, \beta_{2}=1.33$ (E) and $\beta_{2}=2.4$ (F). The travelling wave regime with constant velocity is found independent of the initial condition in the (ID) and (OD) regimes as well.

part of Eq.(37), written in the form $N_{0}=\exp [S(z)]$, and performing a WKB approximation 
$\left(\left|S^{\prime}(z)\right| \gg 1[61]\right)$, we get the following expression for the approximate solution of Eq.(36):

$$
N_{0}(z) \approx \beta_{2} \int_{z}^{0} \frac{\sinh \left(S(z)-S\left(z^{\prime}\right)\right)}{S^{\prime}\left(z^{\prime}\right)} d z^{\prime}+\frac{\beta_{2}}{2\left(\beta_{1} \phi_{e}+\beta_{2}\right)} e^{S(z)}+\left(1-\frac{\beta_{2}}{2\left(\beta_{1} \phi_{e}+\beta_{2}\right)}\right) e^{-S(z)}
$$

with $S(z)=\int_{z}^{0} \sqrt{\beta_{1} \phi_{0}+\beta_{2}} d z^{\prime}$. In particular, it allows an estimation both of the penetration length of the oxygen through the surrounding tissue, and of the size of the proliferating region, expressed as:

$$
l_{p} \sim \frac{\sqrt{\beta_{1} \phi_{e}+\beta_{2}}}{\beta_{1} \phi_{e}}
$$

\subsubsection{Stability analysis of the planar front}
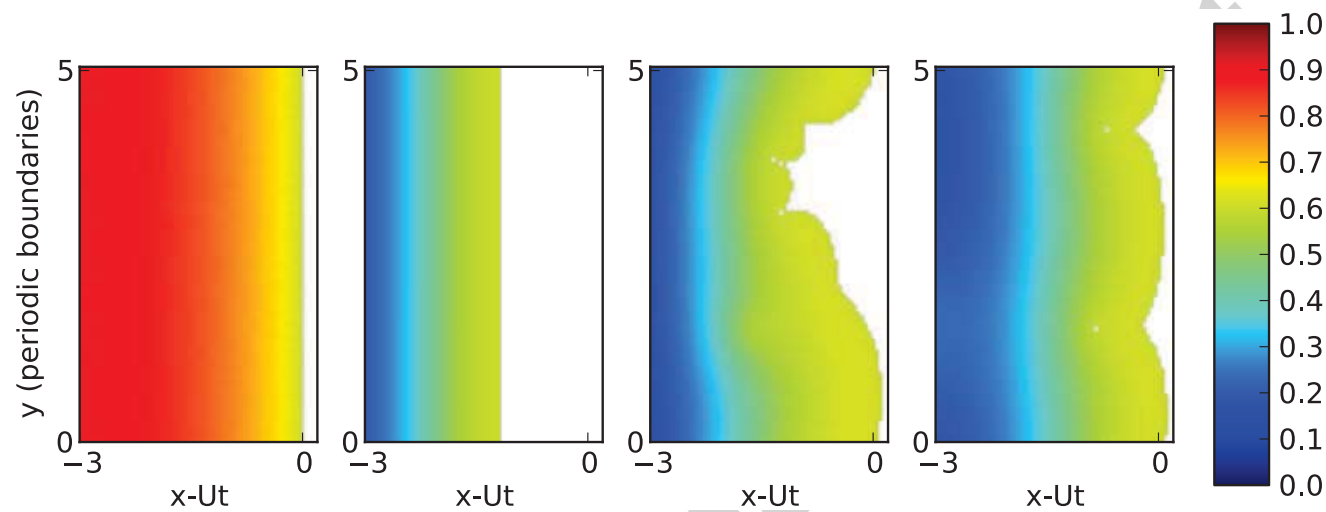

Figure 6: Cell volume fraction $\phi$ during an instability in the (OD) regime occurring on a planar front, propagating along $x$, initially invariant along $y$ and periodic along $y$ axis above instability threshold. The numerical simulations describe a front instability with characteristic finite wavelength $\Lambda$ and stabilization of the pattern at long times. Dimensionless parameters are $\beta_{1}=4, \beta_{2}=0, \phi_{e}=0.6, \delta=0.4$, and $t=0,10,20,80$ from left to right.

For some range of parameters, in numerical simulations we observe the development of an instability, along the y axis, which grows and saturates up to a certain size (Fig.6). An infinitesimal front perturbation with wavelength $\Lambda=2 \pi / \kappa$ inducing a perturbation of $\phi, N$ and $I$ can be written as:

$$
\begin{aligned}
& L(t, y)=L_{0}(t)+\epsilon \cos (\kappa y) e^{\lambda t} \\
& \phi(x, y, t)=\phi_{0}(z)+\epsilon f(z) \cos (\kappa y) e^{\lambda t} \\
& I(x, y, t)=I_{0}(z)+\epsilon p(z) \cos (\kappa y) e^{\lambda t} \\
& N(x, y, t)=N_{0}(z)+\epsilon g(z) \cos (\kappa y) e^{\lambda t}
\end{aligned}
$$

with $\epsilon \ll 1$. Introducing this perturbation in Eqs. $(31,34,33)$ we get at the order $\epsilon$ the following relations:

$$
\begin{aligned}
& \lambda f-U f^{\prime}+\kappa^{2} \phi_{0} K(\phi) \Sigma_{\phi} f-\left(\phi_{0} K(\phi) \Sigma_{\phi} f\right)^{\prime \prime}-\Gamma_{\phi} f=\Gamma_{N} g+\Gamma_{I} p \\
& -\kappa^{2} p+p^{\prime \prime}+\alpha p=-f \\
& -\kappa^{2} g+g^{\prime \prime}-\beta_{1} \phi_{0} g-\beta_{2} g=\beta_{1} N_{0} f \\
& \quad 14
\end{aligned}
$$


with the boundary conditions $f(0)=-\phi_{0}^{\prime}(0)(\mathrm{B} 1), g(0)=-N_{0}^{\prime}, p(0)=-I_{0}^{\prime}(0)$ and $K(\phi) \Sigma_{\phi}\left(f^{\prime}(0)+\right.$ $\left.\phi_{0}^{\prime \prime}\right)=-\lambda(\mathrm{B} 2)$. Notice that the perturbation is not symmetric, so that Eq.(19) is no longer sufficient to solve our system of equations. A closure problem arises again when perturbing the symmetrical solution, as the first-order solution has two additional variables (the two tangential components of the phase velocities) while only one extra equation is given from Eq.(13): one constitutive mobility assumption is therefore necessary. Although many different behaviors might be assumed fulfilling obvious thermodynamic restrictions (e.g. chemotactic/haptotactic mobility in potential flow models for cells [55], or time-correlated molecular motions in entangled systems [62], we assume that $V_{m i x}=0$ even at first order. This is a physically-consistent assumption for a highly viscous system with negligible inertia, because it imposes that the perturbation alone cannot introduce a movement of the center of mass of the mixture in absence of external forces. The long wavelength limit of a system similar to the (OD) regime with $\beta_{2}=0$ was studied by Ciarletta et al. [63]. This analysis gives the parameter range for the stability of travelling wave solutions against long wavelength perturbations. However we found numerically that the instabilities usually appear before entering the unstable domain of long wavelength, suggesting for $\lambda$ the scenario sketched in Fig.7. This is also coherent with the numerical observations of a saturation of the amplitude and the definition of a typical finite wavelength of induced contour undulations in Fig.6 and Fig.10, as also observed in clinical dermatology [64, 65].

Using the change of function $\hat{f}=\exp \left(-\int_{z}^{0} U /(2 G) d z^{\prime}\right) G f$, with $G=\phi_{0} K(\phi) \Sigma_{\phi}$, the Eq.(46) can be formally rewritten as:

$$
\begin{aligned}
& \hat{f}^{\prime \prime}-\left(\kappa^{2}+\zeta_{\lambda}(z)\right) \hat{f}=\left(\Gamma_{I} p+\Gamma_{N} g\right) e^{-\int_{2}^{0} U /(2 G) d z^{\prime}} \approx 0 \\
& \zeta_{\lambda}=\frac{U G^{\prime}}{2 G^{2}}+\frac{1}{4}\left(\frac{U}{G}\right)^{2}-\frac{\Gamma_{\phi}-\lambda}{G}
\end{aligned}
$$

where $\Gamma_{i}=d \Gamma / d i$ for $i=\phi, I, N$. We neglected here the terms in the right hand side of Eq.(46). Such an approximation is exact for the (CI) regime and is justified in the (ID) and (OD) regimes by the large values taken by the function $\zeta_{\lambda}$ (see Fig.7B) if compared to $\Gamma_{I}$ and $\Gamma_{N}$ (equal to one in our model). Ellem and Kay [35] have shown for example the variation of melanoma proliferation rate with nutrient concentration in a culture medium, from which we can get an overestimate of $\Gamma_{N} \approx 0.3$.

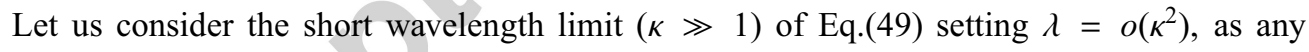
other scalings would be incompatible with the condition (B2). The non diverging WKB solution satisfying the boundary condition (B1) is $\hat{f}(z)=-\phi_{0}^{\prime}(0) \exp (\kappa z)$. At the leading order in $\kappa$, the boundary condition (B2) gives then $\lambda=K(\phi) \Sigma_{\phi} \phi_{0}^{\prime}(0) \kappa$ that can be rewritten as:

$$
\lambda=-U \kappa
$$

For a growing tumor $U>0$ and $\lambda$ is always negative, which means that instabilities cannot develop in the short wavelength domain. On the contrary, for a regressing tumor $U<0$ and, therefore, a short wavelength contour instability always develops during a regression. For melanoma, such regression could be immunologically mediated by $\mathrm{T}$ lymphocytes as found by Halliday et al. [66] or eventually due to a clinical treatment. Regression phenomena are usually associated to scar-like patterns on the tumor contour, as indicated by our analysis. Notice that our macroscopic model is valid only on scales larger than the cell size, typically $6-20 \mu \mathrm{m}$ for melanoma cells [67], and microscopic effects, out of reach for a mixture model, may change the behavior of the eigenvalue $\lambda$ near this size, eventually stabilizing the very short wavelength domain. More- 

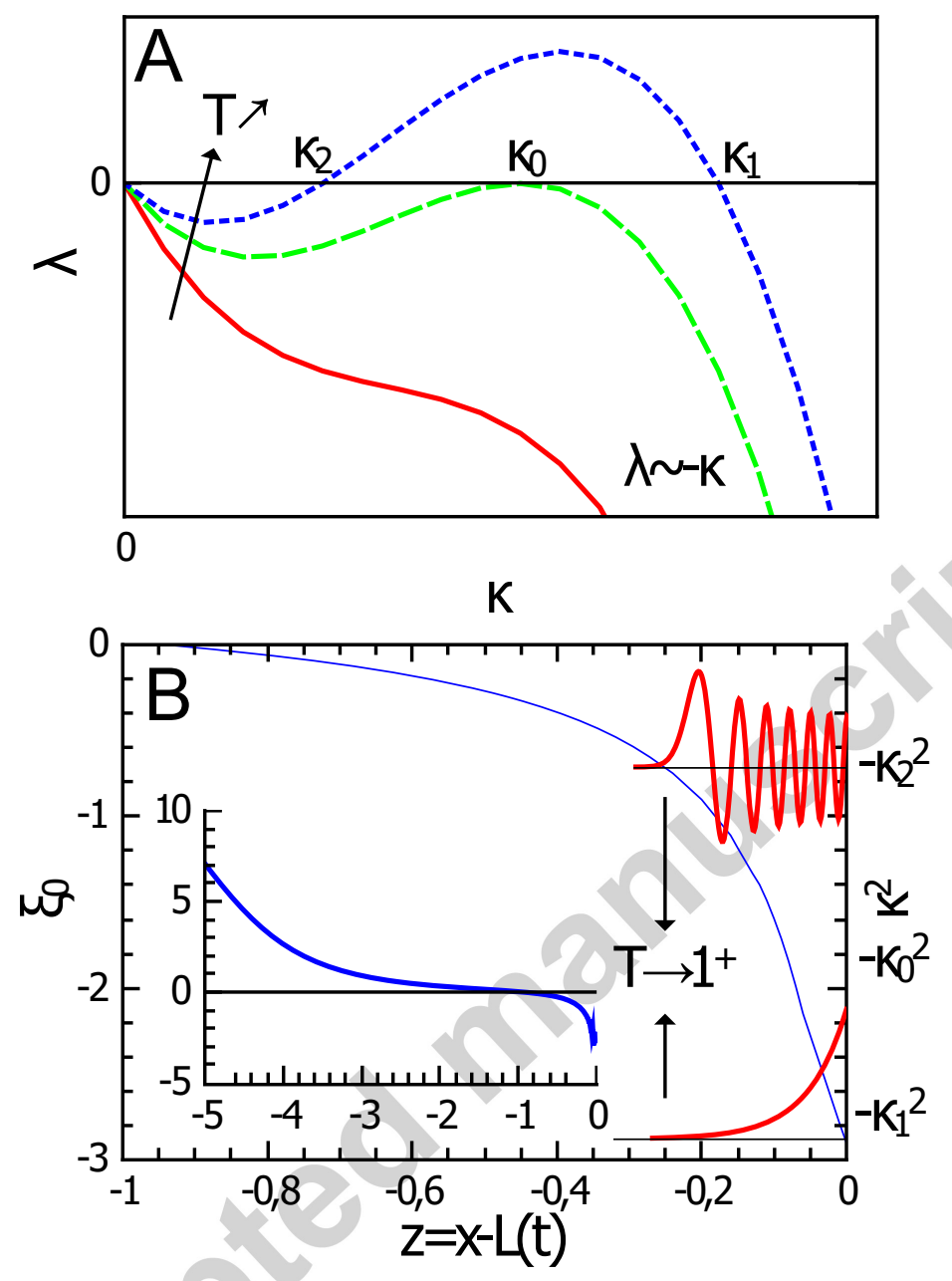

Figure 7: (A) Eigenvalue $\lambda$ as a function of perturbation wavenumber $\kappa$. Analytical results give the behavior near $\kappa=0$ and $\kappa=+\infty$, respectively $\lambda \sim \lambda_{1} \kappa$ and $\lambda \sim-U \kappa^{2}$, with $\lambda_{1}$ the control parameter defined in [63] for a system similar to the (OD) regime. A WKB analysis of the perturbations governing equation gives the existence of two roots $\kappa_{1}>\kappa_{2}$ converging to a single root $\kappa_{0}$ for $T \rightarrow 1^{+}$, where $T$ the control parameter defined in Eq.62. The continuous, dashed and dotted lines sketch the likely variation of the eigenvalue with wavenumber for $T<1$ (stable front), $T=1$ (stability threshold) and $T>1$ (unstable front) respectively. (B) Potential $\zeta_{0}$ from a 1D simulation in the (OD) regime. A WKB analysis of Eq.(49) shows the existence of two solutions satisfying the boundary conditions for a control parameter $T>1$. The first solution with $\kappa_{1}>\kappa_{0}=\sqrt{-\zeta_{0}(0)}$ is decaying exponentially and the second with $\kappa_{2}<\kappa_{0}$ is oscillating until it reaches a turning point at $z_{1}$ with $\zeta_{0}\left(z_{1}\right)=-\kappa_{2}^{2}$. These solutions converge to $\kappa_{0}$ for $T \rightarrow 1^{+}$and disappear for $T<1$. Simulation parameters where $\phi_{e}=0.6, \delta=0.4, \beta_{1}=4$. and $\beta_{2}=1.33$.

over, short wavelength contour undulations are subject to noise [68], being hard to handle for a diagnostic purpose.

In the case of spreading tumors, instabilities should appear first in the domain of finite wavelengths, as suggested by the numerical simulations and in accordance with clinical observations, 
while the instability threshold is formally given by the condition $\lambda=d \lambda / d \kappa=0$, as depicted in Fig.7A. We can now look for the roots of $\lambda(\kappa)$, solving Eq.(49) for $\lambda=0$. Let us first notice that this equation is analog to a Schrödinger equation for a quantum particle with a finite energy $-\kappa^{2}$ in the potential $\zeta_{0}$. This analogy enables a better mathematical understanding of the linear stability analysis. Using the definition of the velocity at the tumor border $z=0$, we get for the potential the following expression:

$$
\zeta_{0}(0)=-\frac{U}{G}\left(\frac{\left(K(\phi) \Sigma_{\phi}\right)_{\phi} U}{2 \phi_{e}\left(K(\phi) \Sigma_{\phi}\right)^{2}}+\frac{U}{4 K(\phi) \Sigma_{\phi} \phi_{e}}+\frac{\Gamma_{\phi}}{U}\right)
$$

In the (ID) and (OD) regimes, we can make the phenomenological assumption that $\Gamma$ is proportional to $\phi$, and the potential $\zeta_{0}$ is therefore negative (for growing tumors) near the tumor border. In the (CI) regime a sufficient condition for $\zeta_{0}(0)<0$ is $\Gamma_{\phi}\left(\phi_{e}\right)>0$ (a necessary and sufficient condition is $\left.\Gamma_{\phi}\left(\phi_{e}\right)>-\left(\phi_{0}^{\prime} / 2\right)^{2}\left(2\left(K(\phi) \Sigma_{\phi}\right)_{\phi}+K(\phi) \Sigma_{\phi} / \phi_{e}\right)\right)$. If the cell volume fraction is low enough in the tumor center $\left(\Phi_{0}<\phi *\right)$, there exists a point $z *$ where $\Sigma_{\phi}$ cancels and $\zeta_{0}$ diverge, acting as centrifugal barrier. As discussed in Section 2.1, this situation can eventually lead to a spinodal decomposition, anyway occuring for specific initial conditions and parameter values, which do not correspond to the objectives of this work. Finally, an asymptotic analysis in $z \rightarrow-\infty$ gives $\zeta_{0} \sim(U / 2 G)^{2}-\Gamma_{\phi} / G$. Moreover $\Gamma_{\phi} \rightarrow 0$ in the (ID) and (OD) regime and $\Gamma_{\phi} \rightarrow \Gamma_{\phi}\left(\phi_{i n h i b}\right)<0$ in the (CI) regime such that $\zeta_{0}$ is strictly positive for $z \rightarrow-\infty$ and negative only on a finite sized domain $z>z_{1}$ near the tumor border. From quantum mechanics we know that the spectrum of eigenvalues $\kappa^{2}$ for such potentials is discrete and finite. This spectrum should converge to a single finite eigenvalue $\kappa_{0}^{2}$ at the instability threshold.

In order to account for the boundary conditions of $\hat{f}$ at $z=0$, let us first write Eq.(35) at the tumor border as:

$$
-U \phi_{0}^{\prime}-\left(K(\phi) \Sigma_{\phi} \phi_{0} \phi_{0}^{\prime \prime}+K(\phi) \Sigma_{\phi} \phi_{0}^{\prime 2}+\left(K(\phi) \Sigma_{\phi}\right)_{\phi} \phi_{0} \phi_{0}^{\prime 2}\right)=\Gamma
$$

Using the expression of the velocity at the tumor border, $-U=K(\phi) \Sigma_{\phi} \phi_{0}^{\prime}$, we can further simplify the previous expression as:

$$
\frac{\phi_{0}^{\prime \prime}}{\phi_{0}^{\prime}}=\frac{\Gamma}{U \phi_{0}}-\frac{\left(K(\phi) \Sigma_{\phi}\right)_{\phi} \phi_{0}^{\prime}}{K(\phi) \Sigma_{\phi}}
$$

Recalling the definition $\hat{f}=\exp \left(-\int_{z}^{0} U /(2 G) d z^{\prime}\right) G f$, its differentiation reads:

$$
\frac{\hat{f}^{\prime}}{\hat{f}}=\frac{U}{2 G}+\frac{G^{\prime}}{G}+\frac{f^{\prime}}{f}
$$

Substituting the expression of $G$ and of the velocity at the tumor border, we get $U /(2 G)=$ $-\phi_{0}^{\prime} /\left(2 \phi_{0}\right)$ and $G^{\prime} / G=\phi_{0}^{\prime} / \phi_{0}+\left(K(\phi) \Sigma_{\phi}\right)_{\phi} \phi_{0}^{\prime} /\left(K(\phi) \Sigma_{\phi}\right)$. The boundary condition with $\lambda=0$ is given by $f^{\prime} / f=\phi_{0}^{\prime \prime} / \phi_{0}^{\prime}$, so that using Eqs. $(54,55)$ we can write the boundary condition for $\hat{f}$ as:

$$
\frac{\hat{f}^{\prime}}{\hat{f}}=\frac{\phi_{0}^{\prime}}{2 \phi_{0}}+\frac{\gamma-\delta}{U}
$$

Let us look for WKB solutions $\hat{f}=A^{+} \exp (S(z))+A^{-} \exp (-S(z))$ with the WKB phase $S^{\prime}(z)^{2}=$ $\kappa^{2}+\zeta_{0}(z)$. This is a typical method of Quantum Mechanics for finding eigenvalues and eigenstates of the Shrödinger equation for a particule in a potential well, and the following derivation is 

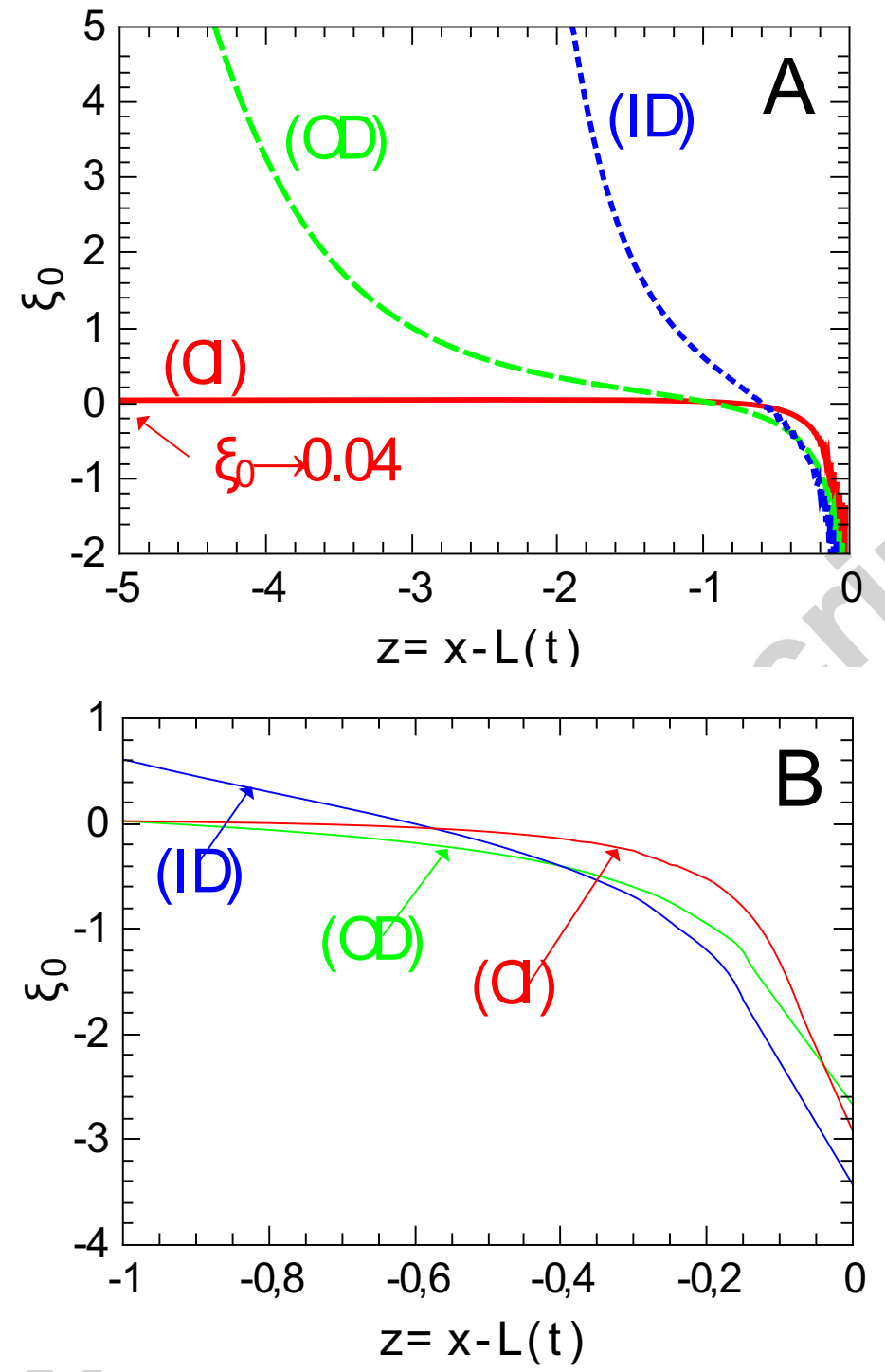

Figure 8: Potential $\zeta_{0}(z)$ in the (CI) regime (red continuous line, $\phi_{\text {inhib }}=0.9$ ), $(\mathrm{OD})$ regime (green dashed line, $\delta=0.4$, $\beta_{1}=4, \beta_{2}=1.32$ ) and (ID) regime (blue dotted line, $\delta=10, \alpha=5$ ). The potential $\zeta_{0}$ is positive at the tumor center $(z \rightarrow-\infty, A)$ and negative near the tumor border $(z=0, \mathrm{~B})$.

similar to standard analysis $[61,69]$. For $\kappa^{2}>\kappa_{0}^{2}=-\zeta_{0}(0), S^{\prime 2}$ is always positive and the non diverging WKB solution is given by:

$$
\hat{f}(z)=\hat{f}(0) \exp \left(-\int_{z}^{0} \sqrt{\kappa^{2}+\zeta_{0}\left(z^{\prime}\right)} d z^{\prime}\right)
$$


This function must satisfy the boundary condition in Eq.(56), formally imposing:

$$
\sqrt{\kappa^{2}+\zeta_{0}(z)}=\frac{\phi_{0}^{\prime}}{2 \phi_{e}}+\frac{\Gamma}{U \phi_{e}}=-\frac{\phi_{0}^{\prime}}{2 \phi_{e}}(T-1)
$$

For a parameter $T=-2 \Gamma /\left(U \phi_{0}^{\prime}\right)>1$ this relation gives a first root $\kappa_{1}$. Notice that the solution $\hat{f}$ corresponding to $\kappa=\kappa_{1}$ vanishes on a typical lengthscale given by $\phi_{0}^{\prime} /\left(2 \phi_{0}\right)(T-1)$, giving also an estimate of the penetration length of the instability inside the tumor. For $\kappa^{2}<\zeta_{0}(0)$, there can be a turning point for $z_{t}>z_{1}$ where the WKB phase vanishes. In the domain $z_{t}<z<0$ the WKB solution is oscillating, and expressed by:

$$
\hat{f}(z)=\hat{f}(0) \frac{\sin \left(S(z)+\psi_{\kappa}\right)}{\sin \left(S(0)+\psi_{\kappa}\right)}
$$

with $S(z)=\int_{z_{t}}^{z} \sqrt{-\kappa^{2}-\zeta\left(z^{\prime}\right)} d z^{\prime}$. In the neighborhood of $z_{t}$, Eq.(49) can be approximated by $\kappa^{2}+\zeta(z)=-a\left(z-z_{t}\right)$, and thus solved in terms of Airy functions. The convergence of the solution for $z \rightarrow-\infty$ imposes a phase angle $\psi_{k}=\pi / 4$. The boundary condition for the nondiverging solution can therefore be expressed as:

$$
\frac{\hat{f}^{\prime}}{\hat{f}}=S^{\prime}(0) \operatorname{cotan}\left(\frac{\pi}{4}+S(0)\right)
$$

The selected $k$ value should thus satisfy the following relation:

$$
\sqrt{-\kappa^{2}-\zeta(0)} \operatorname{cotan}\left(\int_{z_{t}}^{0} \sqrt{-\kappa^{2}-\zeta(z)} d z+\frac{\pi}{4}\right)=-\frac{\phi_{0}^{\prime}}{2 \phi_{0}}(T-1)
$$

The function on the left hand side is vanishing and positive for $\kappa \rightarrow-\zeta_{0}(0)\left(z_{t} \rightarrow 0\right)$ and we conclude that there is at least one solution $\kappa_{2}$ to Eq.(61) for $0<T-1 \ll 1$. The solution $\hat{f}$, corresponding to $\kappa=\kappa_{2}$, is non-zero on a domain whose size is $\left|z_{t}\right|$ and vanishes exponentially for $z<z_{t},\left|z_{1}\right|$ giving a higher bound for $\left|z_{t}\right|$, and therefore for the penetration length of the instability.

We have identified here a parameter $T$ such that, for $T>1$ there exist two distinct eigenvalues $\kappa_{1}>\kappa_{2}$ which are solutions of Eq.(46) for $\lambda=0$, corresponding to the roots depicted in Fig.7. At the stability threshold $T=1$ these eigenvalues converge to $\kappa_{0}$, giving the wavelength of the contour instability as $\Lambda_{0}=2 \pi / \kappa_{0}$. This linear stability analysis is only valid near the stability threshold, however for $T>1$ we expect that the nonlinearities will give a finite amplitude to the contour undulation that should stay stationary, and eventually a correction to the undulation wavelength $\Lambda_{0}$. In particular that the spectrum depicted in Fig.7 is similar to the spectrum of Rayleigh-Bénard instability for which the perturbation amplitude saturates at a finite size [70].

\subsubsection{Growth instability properties of the planar front}

Growth instabilities are therefore controlled at finite wavelength by the parameter $T$, which can be rewritten in physical units as:

$$
T=\frac{2 \Gamma K\left(\phi_{e}\right) \Sigma_{\phi}\left(\phi_{e}\right)}{19^{V_{f}^{2}}}
$$


This expression shows that cell proliferation $(\Gamma)$ and attractive cell-cell interactions $(\Sigma)$ are factors of instability. This finding agrees with earlier results obtained for continuous single-phase models of tumor spheroids and extends it to full multiphase models. Byrne and Chaplain [18] first found an instability driven by competition between an expensive force due to cell proliferation and a restraining force due to cell adhesion. Similarly, Cristini et al. [71] identified two parameters representing the ratio between mitosis and apoptosis and the relative rate of mitosis to the relaxation mechanisms, finding a good agreement with in vitro experiments on glioblastoma tumor spheroids [72]. Our control parameter additionally predicts that slow growing tumors $\left(V_{f}\right)$ are more unstable.

Using the simple scaling $\phi_{0}^{\prime}(0)=-\phi_{e} / l_{p}$, with $l_{p}$ being the size of the proliferating domain, we get the following scaling for the control parameter:

$$
T \sim \frac{2 \Gamma l_{p}}{V_{f} \phi_{e}}
$$

which can be interpreted as the ratio between the mass creation rate in the proliferating area of the tumor and the mass evacuation rate, the contour instability occurring when the proliferation rate is too high, or when the melanoma growth is too slow. The WKB analysis shows that the selected wavenumber $\kappa$ is scaled by $\zeta_{0}$, which is in turn scaled as $\phi_{0}^{\prime} / \phi_{0}$. Therefore, using the same simple scaling, we get an estimation of the instability wavelength as follows:

$$
\Lambda_{0} \sim 2 \pi l_{p}
$$

Finally, the domain $\zeta_{0}<0$ corresponds to the region out of which the term $\Gamma_{\phi} / G$ in $\zeta_{0}$ is negligible or negative, its size being therefore scaled by $l_{p}$. The WKB solution of Eq.(49) shows that the perturbation $\hat{f}$ is vanishing outside this region, meaning that the instability is localized in the outer part of the tumor, within a width comparable to the instability wavelength and to the width of the proliferating domain of the tumor. In a recent study, Dervaux and Ben Amar [73] found a similar result in a poro-elastic growth model, validated by experiments on biomimetic swelling gels.

\subsection{Contour instability of a circular tumor front}

In the more realistic case of a circular tumor front, we observe numericaly that a quiescent (or necrotic) core develops quickly, so that all the cancer activity is confined in a ring of quasiconstant width $l$. Moreover, the tumor radius $R(t)$ grows linearly in time $(d R / d t=U)$ after a transitory regime dependent on the initial conditions, as observed in vivo [48, 74]. Using polar coordinates $(r, \theta)$, Eq. (31) can be rewritten as:

$$
\frac{\partial \phi}{\partial t}-\frac{1}{r} \frac{\partial}{\partial r} r \phi K(\phi) \Sigma_{\phi} \frac{\partial}{\partial r} \phi-\frac{1}{r^{2}} \frac{\partial}{\partial \theta} \phi K(\phi) \Sigma_{\phi} \frac{\partial}{\partial \theta} \phi=\Gamma(\phi, N, I)
$$

We can perform the conformal transformation $z=R \ln (r / R), y=R \theta$ and look for adiabatic solutions $\phi_{R}(t, z, y)$ of the transformed equation, expressed as:

$$
\frac{\partial \phi_{R}}{\partial t}-U\left(1-\frac{z}{R}\right) \phi_{R}^{\prime}-e^{-2 z / R}\left(\phi_{R} K\left(\phi_{R}\right) \Sigma_{\phi} \phi_{R}^{\prime}\right)^{\prime}-e^{-2 z / R} \frac{\partial}{\partial y} \phi_{R} K\left(\phi_{R}\right) \Sigma_{\phi} \frac{\partial}{\partial y} \phi_{R}=\Gamma
$$

which is very similar to Eq.(46) for the planar case. For the sake of simplicity, we drop the indice $R$ in the following. 

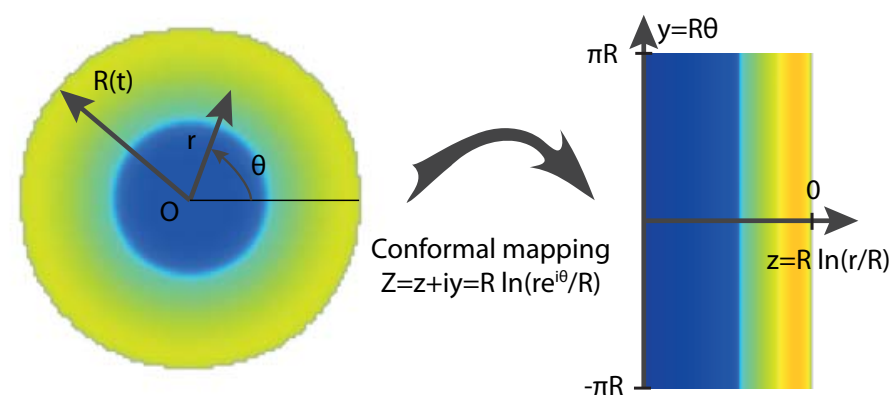

Figure 9: After a conformal mapping $Z=z+i y=R \ln \left(r e^{i \theta} / R\right)$, the radial growth is transformed into a planar growth invariant along the $y$-axis and propagating from $z=-\infty$ along a $x$-axis in the moving frame $z=x-L(t)$, with the tumor border $L(t)$

\subsubsection{Linear stability analysis of the circular front}

From the numerical simulations depicted in Fig.10, we acknowledge that a radially symmetric solution $\phi_{0}(z)$ exists, satisfying $\partial \phi_{0} / \partial t=\partial \phi_{0} / \partial y=0$ in Eq.(66). Such a solution is also unstable for some range of the physical parameters. Considering a contour perturbation with wavelength $2 \pi / \kappa$ along $y=R \theta$, the governing equation can be rewritten as:

$$
\lambda f-U\left(1-\frac{z}{R}\right) f^{\prime}-D e^{-2 z / R}\left(\phi_{0} K(\phi) \Sigma_{\phi} f\right)^{\prime \prime}+D \kappa^{2} e^{-2 z / R} \phi K(\phi) \Sigma_{\phi} f-\Gamma_{\phi} f=\Gamma_{N} g+\Gamma_{I} h
$$

with the boundary conditions given by $K(\phi) \Sigma_{\phi}\left(\phi_{0}^{\prime \prime}(0)-\phi_{0}^{\prime} / R+f^{\prime}(0)\right)=-\lambda$ and $f(0)=-\phi_{0}^{\prime}(0)$. Considering the change of function $\hat{f}=G \exp \left(-\int_{z}^{0} U\left(1-z^{\prime} / R\right) e^{2 z^{\prime} / R} /(2 G) d z^{\prime}\right) f$ with $G=$ $\phi_{0} K(\phi) \Sigma_{\phi}$, we get an equation similar to Eq.(49), defining a potential $\zeta_{\lambda, R}(z)$ as:

$$
\zeta_{\lambda, R}(z)=\frac{U\left(1-\frac{z}{R}\right) G^{\prime} e^{2 z / R}}{2 G^{2}}+\frac{U\left(1-\frac{2 z}{R}\right) e^{2 z / R}}{2 R G}+\frac{1}{4}\left(\frac{U\left(1-\frac{z}{R}\right) e^{2 z / R}}{G}\right)^{2}-\frac{\Gamma_{\phi}-\lambda}{G} e^{2 z / R}
$$

In the short wavelength limit $(\kappa \gg 1)$, the equation for $\hat{f}$ is the same as the planar case, and the solution is $\hat{f}(z)=-\phi_{0}^{\prime}(0) \exp (\kappa z)$, leading to $\lambda=K(\phi) \Sigma_{\phi} \phi_{0}^{\prime}(0) \kappa=-U \kappa<0$. As expected, stability properties in this wavelength domain are unchanged, and only regressing tumors are unstable.

Looking for $\kappa$ with $\lambda=0$, we need to solve Eq.(49) with the potential $\zeta_{0, R}$ given in Eq.(68), and we can perform the same analysis as for the planar case. Let us first notice that $\zeta_{0, R}(z)$ is a decreasing function of $R$, which is large and positive at the beginning of the growth $\left(\zeta_{0, R}(z) \sim 1 / R\right.$ for $R \ll 1)$ and negative close to the tumor border when $R$ exceeds a critical radius $1 / R_{c}=$ $2(\gamma-\delta) / U-G^{\prime} / G-U /(2 G)$, so that we can write $\zeta_{0, R}(0)=(U /(2 G))\left(1 / R-1 / R_{c}\right)$. As for the planar case, the circular front become unstable if it exits $\kappa>0$ satisfying the following relation:

$$
\sqrt{\kappa^{2}+\zeta_{0, R}(0)}=-\frac{\phi_{0}^{\prime}}{2 \phi_{0}}(T-1)-\frac{1}{R}
$$

A necessary condition is therefore given by $1 / R<-\phi_{0}^{\prime} /\left(2 \phi_{0}\right)(T-1)=1 / R_{e}$, with:

$$
-\frac{\phi_{0}^{\prime}}{2 \phi_{0}}(T-1)=(\gamma-\delta) / U+\phi_{0}^{\prime} /\left(2 \phi_{0}\right)
$$



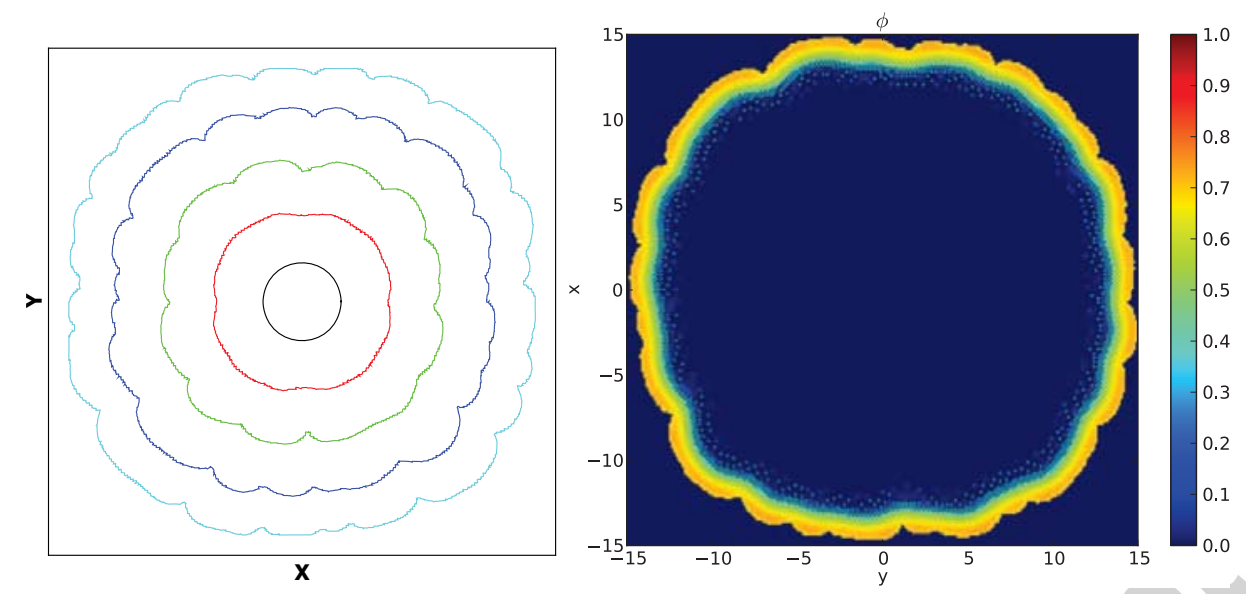

Figure 10: Contour instability occurring during the radial growth of a tumor initially circular in the (OD) regime. (A) Tumor contour at time $t=0, t=10, t=20, t=30$ and $t=40$ from smaller to larger diameter. Notice the definition of a typical wavelength. (B) Cell volume fraction $\phi$ at $t=40 . \beta_{1}=4, \beta_{2}=0, \phi_{e}=0.6, \delta=0.4$

The sign of $\zeta_{0, R}$ is given by $1 / R-1 / R_{c}$, so that:

$$
\frac{1}{R_{c}}=2 \frac{\gamma-\delta}{U}-\frac{\phi_{0}^{\prime}}{2 \phi_{0}}-\frac{W_{\phi, \phi} \phi_{0}^{\prime}}{W_{\phi}}>\frac{1}{R_{e}}
$$

showing that $\zeta_{0, R}$ is negative for $R>R_{e}>R_{c}$ and that it exists a $\kappa>0$ satisfying Eq.(69). We conclude that the circular front becomes unstable only after that the tumor size reaches a threshold radius $R_{e}$, which becomes infinite when $T \rightarrow 1^{+}$. For large $T$, let us remind that this analysis holds only after the steady growth regime is reached, typically after the development of a quiescent or necrotic core, and therefore the tumor radius must be larger than the proliferating domain size $l_{p}$.

\subsubsection{Growth instability properties of the circular front}

Recalling the simple scaling $\phi_{0} / \phi_{0}^{\prime} \sim l_{p}$, we can get an estimate of the threshold radius as follows:

$$
R_{e} \sim \frac{2 l_{p}}{T-1}
$$

In particular the phase diagram in Fig. 11 shows the stability of the tumor contour as a function the tumor radius and of the ratio between the tumor growth rate and the tumor cell proliferation at the tumor border. Furthermore, the expression of $\zeta_{0, R}(0)$ shows that the instability wavelength $\Lambda$ is a decreasing function of $R$, with $\Lambda \gg 1$ for $R \gtrsim R_{e}$ and $\Lambda_{0} \sim 2 \pi l_{p}$ for $R \ll R_{e}$. Again, these results are in agreement with the biomimetic growth model of Dervaux and Ben Amar [73]. Indeed, in their study they found that the tumor becomes more unstable when the ratio between the size of the proliferating ring and the outer radius decreases, which appears in our model through the existence of a threshold radius. Their control parameter corresponds to the amount of growth in the proliferating ring and can be estimated as $\Gamma \tau_{c}$, where $\tau_{c}=l_{p} / V_{f}$ is the characteristic time of evacuation of the created mass. This leads a control parameter of about $\Gamma l_{p} / V_{f}$, very similar 


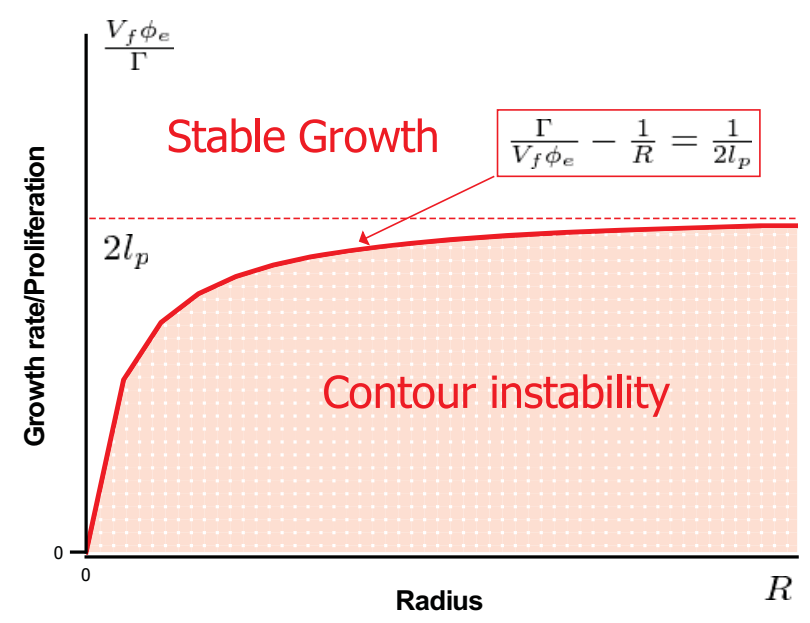

Figure 11: Stability of the radial growth as a function of tumor radius and of the ratio between the tumor growth rate $V_{f}$ and the mean tumor cell proliferation at the tumor border $\Gamma / \phi_{e}$. Regressing tumors with $V_{f}<0$ are always unstable.

\begin{tabular}{|c|c|c|}
\hline Morphology & Stable contour & Unstable contour \\
\hline Control parameter $T$ & $<1$ & $>1$ \\
\hline Tumor radius $R$ & $<R_{e}$ & $>R_{e}(\Lambda$ decreases with $R)$ \\
\hline cell-cell interactions $\Sigma$ & weak attraction & strong attraction $\left(\Lambda \sim l_{p}\right)$ \\
\hline cell proliferation $\Gamma$ & low & high $\left(\lambda \sim l_{p}\right)$ \\
\hline tumor growth rate $V_{f}$ & fast progression & $\begin{array}{c}\text { slow progression }\left(\Lambda \sim l_{p}\right) \\
\text { regression }\left(\Lambda \ll l_{p}\right)\end{array}$ \\
\hline
\end{tabular}

Table 3: Stability properties of the tumor contour in our two-phase model. Correlation with the tumor cells phenotype, e.g. mechanical interactions $(\Sigma)$ and proliferation rate $(\Gamma)$, and macroscopic tumor properties, e.g. growth rate $\left(V_{f}\right)$ and radius $(R)$.

to the one found in our analysis, while the threshold has the same qualitative dependance on the tumor radius. Pham et al. [75] also found a similar behavior in a single phase model, with a control parameter corresponding to cell adhesion and a stability threshold decreasing with the tumor radius.

\section{Discussion and comparison with clinical observations}

In this work, we have proved the existence of contour instabilities in a mixture model of the early growth of melanoma, using both analytical and numerical techniques. The proposed multiphase model considers the influence of cancer cells proliferation/inhibition mechanisms (nutrient diffusion, contact inhibition, endocrine/paracrine regulations) as well as of the mechanical interaction between cells.

In Section 2 we have derived a 2D multiphase model in the lubrication limit of a 3D model, with governing equation expressed by Eqs. $(31,32,33,34)$, taking into account the thin geometry 


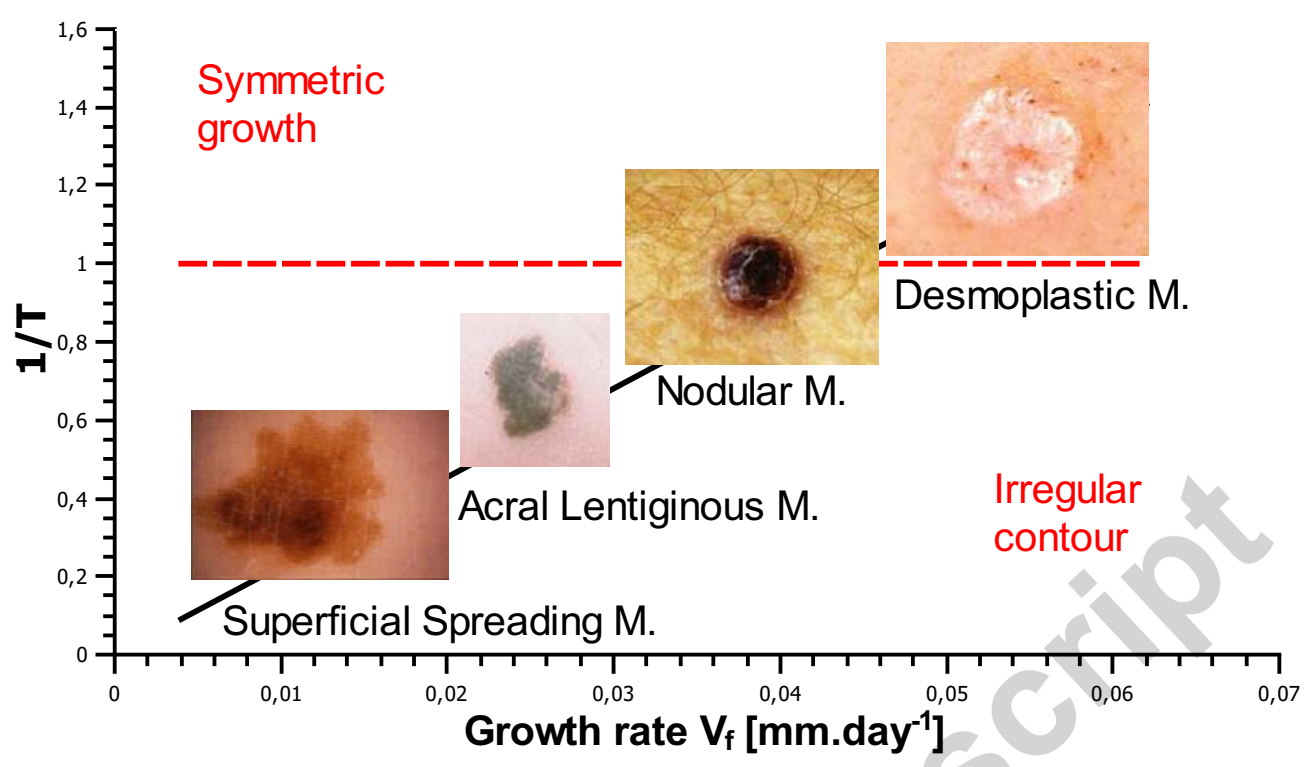

Figure 12: Control parameter $T$ estimated from Eq.63 for slow growing melanoma (Superficial Spreading and Acral Lentiginous types) and fast growing melanoma (Nodular and Desmoplastic types). Median tumor growth rate are taken from [48] and model parameters are reported in table 1. Fast growing tumors with $T<1$ are characterized by a symmetrical radial growth while the fast growing tumors with $T>1$ are usually asymmetric. Images are a courtesy of Dr. Pascale Guitera (Superficial Spreading Melanoma), the Skin Cancer Foundation [76] (Acral Lentigunous Melanoma) and the Cliveland clinic [77] (Nodular and Desmoplastic Melanoma).

of the epidermis. The physical values for the model parameters have been extracted from experimental results in related literature, and reported in Table 1. In Section 3.1.1, the numerical simulations with given scaling have shown that the tumor cells proliferate only on an external domain of size $l_{p}$, which tends to decrease as tumor cell keep on uptaking the diffusing nutrients, even if both the basement membrane and stratum corneum are permeable to nutrient and growth inhibitor, as shown in Eqs. $(39,41)$. In Sections 3.1.3 and 3.2.2, we have identified the stability properties of the melanoma contour in our model for both planar and circular fronts. As summarized in Table 3, regressing melanoma (negative growth rate) have always an unstable border with short wavelength undulations (Eq.(51)), while spreading melanoma present a finite wavelength contour instability when the control parameter $T$ is larger than 1 (Eq.(62)) and the tumor radius exceeds a critical value (Eqs.(69,72)). In particular, high cell-to-cell adhesion, high cell proliferation and slow tumor growth are factors of instability, and the resulting contour undulation $\Lambda$ has a size comparable to the extend $l_{p}$ of the outer proliferating domain (Eq.(64)). The experimental data collected in Table 1 give in the (OD) regime typical values of $\Lambda=0.43-2.2$ $\mathrm{mm}$, which are compatible with the clinical observations and with the mixture model hypothesis, the typical melanoma cell size being $6-20 \mu \mathrm{m}$ as reported by Clark et al.[67]. Our results agree with earlier results on single phase tumor models $[18,71,72]$, while applying to a multiphase mixture. The stability of the tumor front is determined by a competition between the cell proliferation $\Gamma$ and cell adhesion $\Sigma$, where stronger adhesion between cells promotes the occurrence of 


\begin{tabular}{|c|c|c|}
\hline $\begin{array}{c}\text { Growth rate } \\
{\left[\mathrm{mm} \mathrm{day}^{-1}\right]}\end{array}$ & Control parameter $T$ & $\begin{array}{c}\text { Radius threshold } R_{e} \\
{[\mathrm{~mm}]}\end{array}$ \\
\hline $0.004($ median SSM $)$ & $11-18$ & $0.021-0.022$ \\
\hline 0.011 (SSM ) & $4-6.5$ & $0.065-0.073$ \\
\hline 0.0043 (median LMM ) & $10-17$ & $0.023-0.024$ \\
\hline 0.013 (median ALM) & $3.4-5.5$ & $0.079-0.092$ \\
\hline 0.016 (median NM ) & $2.75-4.5$ & $0.1-0.13$ \\
\hline 0.049 (fast growing NM ) & $0.9-1.5$ & $>0.77$ \\
\hline 0.062 (fast growing DM ) & $0.71-1.2$ & $>2.2$ \\
\hline
\end{tabular}

Table 4: Abbreviations: superficial spreading melanoma (SSM), lentigo maligna melanoma (LMM), acral lentiginous melanoma (ALM), nodular melanoma (NM) and desmoplastic melanoma (DM). The parameter $T$ is estimated from Eq.63 in the (OD) regime and $R$ from Eq.72. The growth rates are reported from $[48,78]$ and the model parameter are $\gamma=0.2 \mathrm{day}^{-1}$ and $l_{p}=0.11-0.18 \mathrm{~mm}$ as reported in table 1 .

contour undulations.

Interestingly, our control parameter $T$ indicates a strong correlation between the melanoma growth rate and the stability of its contour. In particular, theoretical predictions indicate that fast growing melanoma are more stable than slow growing ones, while regressing melanoma (negative growth rate) are expected to present short wavelength contour instabilities. Indeed these results agree with the studies of Liu et al. [48] and Argenziano et al. [78], focused on fast and slow growing melanoma, respectively. Using such results, in Table 4 we can estimate the value of our control parameter for each growth rate, taking into account the reported experimental values for the model parameters (see Table 1 ). We typically find $T<1$ for fast growing melanoma (i.e. $V_{f}>1.5 \mathrm{~mm}$ per month, for nodular melanoma), and $T>1$ for slow growing melanoma $\left(V_{f}<\right.$ $0.4 \mathrm{~mm}$ per month), in agreement with the observed morphology of such tumors, respectively possessing more and less regular contours [48]. We plot in Fig.12 the inverse of this parameter in function of the growth velocity for different kinds of melanoma.

The combination of our theoretical results and clinical data confirms that general undulations of the perimeter, known as structure irregularities, may infer the abnormal important histological signs and have a high correlation with melanomas [68], in opposition with smaller wavelength texture irregularities. Therefore, the extent of our findings suggests that short-term follow-up of pigmented skin lesions may be a promising approach to recognize malignant lesion as early as possible. The correlation of the results from theoretical modeling and simulations tools with dermoscopy/histopathology data of primary melanomas can eventually drive progress to assess early diagnostic rules in the short-term clinical follow-up of pigmented skin lesions.

Avascular melanoma is the ideal system model for the theoretical study of tumor development, because its radial growth phase can be widely investigated using a simplified twodimensional model, both with analytical and numerical techniques. Furthermore, a large amount of histopathological and clinical data are available in clinical dermatology, allowing validation of the theoretical predictions with respect to the morphological evolution of different skin lesions. This work represents a first insight in the multiphase description of such a system model, opening novel perspectives for future studies concerning the relations between tumor microstructure, cell phenotypes and contour morphology. 


\section{Acknowledgements}

We thank Dr H. Kittler and Dr P. Guitera for giving us access to clinical data.

\section{References}

[1] R. S. Stern, Prevalence of a history of skin cancer in 2007: results on a incidence-based model, Arch. Dermatol. 146 (2010) 279-282.

[2] M. A. Weinstock, Cutaneous melanoma: public health approach to early detection, Dermatol. Ther. 19 (2006) 26-31.

[3] P. R. Guitera, New imaging methods for the diagnosis of melanoma and its metastasis, Ph.D. thesis, Faculté de médecine Paris Sud (2009).

[4] E. Farmer, R. Gonin, M. Hanna, Discordance in the histopathologic diagnosis of melanoma and melanocytic nevi between expert pathologists, Human Path. 27 (1997) 528-531.

[5] T. K. Lee, D. I. McLean, M. S. Atkins, Irregularity index: A new border irregularity measure for cutaneous melanocytic lesions, Med. Image Anal. 7 (2003) 47-64.

[6] H. Kittler, et al., Identification of clinically featureless incipient melanoma using sequential dermoscopy imaging, Arch. Dermatol. 142 (2006) 1113-1119.

[7] C. Dolianitis, J. Kelly, R. Wolfe, P. Simpson, Comparative performance of 4 dermoscopic algorithms by nonexperts for the diagnosis of melanocytic lesions, Arch. Dermatol. 141 (2005) 1008-1014.

[8] R. K. Amaravadi, K. T. Flaherty, Targeted therapy for metastatic melanoma, Clinical Adv. Hem. Onc. 5 (2007) 386-394.

[9] F. S. Hodi, et al., Improved survival with ipilimumab in patients with metastatic melanoma, New Eng. J. Med. 363 (2010) 711-723.

[10] H. Skvara, L. Teban, M. Fiebiger, M. Binder, H. Kittler, Limitations of dermoscopy in the recognition of melanoma, Arch. Dermatol. 141 (2005) 155-160.

[11] T. B. Fitzpatrick, A. S. Breathnach, The epidermal melanin unit system, Dermatol. Wochenschr. 147 (1963) $487-$ 489.

[12] N. K. Haass, M. Herlyn, Normal human melanocyte homeostasis as a paradigm for understanding melanoma, J. Invest. Dermatol. Symp. Proc. 10 (2005) 153-163.

[13] L. Larue, F. Beermann, Cutaneous melanoma in genetically modified animals, Pigment Cell Res. 20 (2007) 485497.

[14] E. Lázár-Molnár, H. Hegyesi, S. Tóth, A. Falus, Autocrine and paracrine regulation by cytokines and growth factors in melanoma, Cytokine 12 (2000) 547-554.

[15] V. Cristini, et al., Morphologic instability and cancer invasion, Clin. Cancer Res. 11 (2005) 6772.

[16] S. Eikenberry, C. Thalhauser, Y. Kuang, Tumor-immune interaction, surgical treatment, and cancer recurrence in a mathematical model of melanoma, PLoS Comput. Biol. 5 (2009) e1000362.

[17] J. R. King, S. J. Franks, Mathematical Modeling of Biological Systems, Birkhauser Edition, Vol. 1, A. Deutsch, L. Brusch, H. Byrne, G. Vries and H. Herzel, 2007, Ch. Stability properties of some tissue-growth models, p. 175.

[18] H. M. Byrne, M. A. J. Chaplain, Free boundary value problems associated with the growth and development of multicellular spheroids, Euro. J. Appl. Math. 8 (1997) 639-658.

[19] J. S. Lowengrub, et al., Nonlinear modelling of cancer: Bridging the gap between cells and tumours, Nonlinearity 23 (2010) 1-91.

[20] P. J. Murray, C. M. Edwards, M. J. Tindall, P. K. Maini, From discrete to continuum model of cell dynamics in one dimension, Phys. Rev. E 80 (2009) 031912.

[21] P. Tracqui, Biophysical models of tumour growth, Rep. Prog. Phys. 72 (2009) 056701-30.

[22] A. R. A. Anderson, V. Quaranta, Integrative mathematical oncology, Nature Rev. Cancer 8 (2008) 227-234.

[23] L. Graziano, L. Preziosi, Modeling of biological materials, Birkhauser Edition, F. Mollica, L. Preziosi and K. R. Rajagopal, 2007, Ch. Mechanics in tumor growth, pp. 263-313.

[24] C. J. W. Breward, H. M. Byrne, C. E. Lewis, A multiphase model describing vascular tumour growth, Bull. Math. Biol. 65 (2003) 609-640.

[25] A. Ambrosi, L. Preziosi, Cell adhesion mechanisms and stress relaxation in the mechanics of tumours, Biomech. Model. Mechanobiol. 8 (2009) 397-413.

[26] V. Cristini, X. Li, J. S. Lowengrub, S. M. Wise, Nonlinear simulations of solid tumor growth using a mixture model: invasion and branching, J. Math. Biol. 58 (2009) 723-763.

[27] S. Astanin, L.Preziosi, Selected Topics in Cancer Modeling, Birkhauser Edition, N. Bellomo, M. Chaplain and E. de Andelis, 2008, Ch. Multiphase models of tumour growth, p. 1. 
[28] M. Ben Amar, C. Chatelain, P. Ciarletta, Contour instabilities in early tumor growth models, Phys. Rev. Lett., in press.

[29] H. Wiig, R. K. Reed, O. Tenstad, Interstitial fluid pressure, composition of interstitium, and interstitial exclusion of albumin in hypothyroid rats, Am. J. Physiol. Heart Circ. Physiol. 278 (2000) 1627-1639.

[30] R. K. Jain, Transport of molecules in the tumor interstitium: a review, Cancer Res. 47 (1987) 3039-3051.

[31] P. M. van Kemenade, J. M. Huyghe, L. F. A. Douven, Triphasic fe modeling of skin water barrier, Transp. Porous Media 50 (2003) 93-109.

[32] R. Halaban, S. Ghosh, P. Duray, J. M. Kirkwood, A. B. Lerner, Human melanocytes cultured from nevi and melanomas, J. Invest. Dermatol. 87 (1986) 95-101.

[33] L. M. Coussens, Z. Werb, Inflammation and cancer, Nature 420 (2002) 860-867.

[34] P. Vaupel, A. Mayer, Hypoxia in cancer : significance and impact on clinical outcome, Cancer Metastasis Rev. 26 (2007) 225-239.

[35] K. A. O. Ellem, G. F. Kay, The nature of conditioning nutrients for human malignant cultures, J. Cell Sci. 62 (1983) 249-166.

[36] B. Bedogni, M. B. Powell, Hypoxia, melanocytes and melanoma - survival and tumor development in the permissive microenvironment of the skin, Pigment Cell Melanoma Res. 22 (2009) 166-174.

[37] U. Rodeck, et al., Transforming growth factor beta production and responsiveness in normal human melanocytes and melanoma cells, Cancer Res. 54 (1994) 575-581.

[38] K. Krasagakis, C. Grabe, C. C. Zouboulis, C. E. Orfanos, Growth control of melanocytes and melanoma cells by cytokines, Melanoma Res. 3 (1993) 26.

[39] A. A. Creasey, et al., Biological properties of human melanoma cells in culture, In Vitro 5 (1979) 342-350.

[40] G. Helmlinger, P. A. Netti, H. C. Lichtenbeld, R. J. Melder, R. K. Jain, Solid stress inhibits the growth of multicellular tumor spheroids, Nature Biotech. 15 (1997) 778-783.

[41] M. Chaplain, L. Graziano, L. Preziosi, Mathematical modelling of the loss os tissue compression responsiveness and its role in solid tumour development, Math. Med. Biol. 23 (2006) 197-229.

[42] A. Breslow, Thickness, cross-sectional areas and depth of invasion in the prognosis of cutaneous melanoma, Ann. Surgery 5 (1970) 902-908.

[43] H. M. Shaw, W. H. McCarthy, Small-diameter malignant melanoma: a common diagnosis in new south wales, australia, J. Am. A. Dermatol. 27 (1992) 679-682.

[44] M. Stucker, et al., The cuteneous uptake of atmospheric oxygen contributes significantly to the oxygen supply of human dermis and epidermis, J. Physiol. 538 (2002) 985-994.

[45] M. E. Hystad, E. K. Rofstad, Oxygen consumption rate and mitochondrial density in human melanoma monolayer cultures and multicellular spheroids, Int. J. Cancer 57 (1994) 532-537.

[46] B. Bedogni, et al., The hypoxic microenvironment of the skin contributes to akt-mediated melanocyte transformation, Cancer Cell 8 (2005) 443-454.

[47] M. E. Johnson, D. Blankschtein, R. Langer, Evaluation of solute permeation through the stratum corneum: lateral bilayer diffusion as the primary transport mechanism, J. Pharm. Sc. 86 (1997) 1162-1172.

[48] W. Liu, et al., Rate of growth in melanomas, Arch. Dermatol. 142 (2006) 1551-1558.

[49] S. M. Wise, J. S. Lowengrub, H. B. Frieboes, V. Cristini, Three-dimensional multispecies nonlinear tumor growth - I model and numerical method, J. Theor. Biol. 253 (2008) 524-543.

[50] E. A. Swabb, J. Wei, P. M. Gullino, Diffusion and convection in normal and neoplastic tissues, Cancer Res. 34 (1974) 2814-2822.

[51] M. Doi, A. Onuki, Dynamic coupling between stress and composition in polymer solutions and blends, J. Physique II 2 (1992) 1631-1656.

[52] C. A. Kristensen, M. Nozue, Y. Boucher, R. K. Jain, Reduction of interstitial fluid pressure after tnf-alpha treatment of three human melanoma xenografts, British J. Cancer 74 (1996) 533-536.

[53] S. B. Hoath, D. G. Leahy, The organization of human epidermis: functional epidermal units and phi proportionality, J. Invest. Dermatol. 121 (2003) 1440-1446.

[54] Y. Kuwahara, et al., Quantification of hardness, elasticity and viscosity of the skin of patients with systemic sclerosis using a novel sensing device (vesmeter): a proposal for a new outcome measurement procedure, Rheumatology 47 (2008) 1018-1024.

[55] D. Ambrosi, L. Preziosi, On the closure of mass balance models for tumor growth, Math. Mod. Meth. Appl. Sci 12 (2002) 737-754

[56] E. de Angelis, L. Preziosi, Advection-diffusion models for solid tumor evolution in vivo and related free boundary problem, Math. Models Meth. Appl. Sci. 10 (2000) 379-407.

[57] L. I. Cardenas-Navia, et al., Tumor-dependent kinetics of partial pressure of oxygen fluctuations during air and oxygen breathing, Cancer Res. 34 (2004) 6010-6017.

[58] R. Sagebiel, Regression and other factors of prognostic interest in malignant melanoma, Arch. Dermatol. 121 (1985) 1125-1126. 
[59] L. Brogelli, U. M. Reali, S. Moretti, C. Urso, The prognostic significance of histologic regression in cutaneous melanoma, Melanoma Res. 2 (1992) 87-91.

[60] R. A. Zeff, A. Freitag, C. M. Grin, J. M. Grant-Kels, The immune response in halo nevi, JAAD 37 (1997) 620-624.

[61] C. M. Bender, S. A. Orszag, Ch. WKB theory.

[62] P. de Gennes, Scaling concepts in polymer physics, cornell university press Edition, 1979.

[63] P. Ciarletta, L. Foret, M. Ben Amar, The radial growth phase of malignant melanoma: multiphase modeling, numerical simulations and linear stability analysis, J. R. Soc. Interface, in pressdoi:doi:10.1098/rsif.2010.0285.

[64] E. Claridge, P. N. Hall, M. Keefe, J. P. Allen, Shape analysis for classification of malignant melanoma, J. Biomed. Eng. 14 (1992) 229-234.

[65] T. K. Lee, D. I. McLean, M. S. Atkins, Irregularity index: a new border irregularity measure for cutaneous melanocytic lesions, Med. Image An. 7 (2002) 47-64.

[66] G. M. Halliday, A. Patel, M. J. Hunt, F. J. Tefany, R. S. Barneston, Spontaneous regression of human melanoma/nonmelanoma skin cancer: association with infiltrating cd4+ t cells, World J. Surg. 19 (1995) 352-358.

[67] W. H. Clark, L. From, E. A. Bernardino, M. C. Mihm, The histogenesis and biological behavior of primary human malignant melanoma of the skin, Cancer Res. 29 (1969) 705-726.

[68] V. T. Ng, B. Y. Fung, T. K. Leev, Determining the asymmetry of skin lesion with fuzzy borders, Comput. Biol. Med. 35 (2005) 103-120.

[69] L. D. Landau, L. M. Lifshitz, Quantum Mechanics Non-Relativistic Theory, elsevier science Edition, 1977.

[70] F. Charru, Instabilités hydrodynamiques, edp sciences paris Edition, CNRS, 2007.

[71] V. Cristini, J. S. Lowengrub, Q. Nie, Nonlinear simulation of tumor growth, J. Math. Biol. 46 (2003) 191-224

[72] H. Frieboes, X. Zheng, C. Sun, B. Tromberg, R. Gatenby, V. Cristini, An integrated computational/experimental model of tumor invasion, Cancer Res. 66 (2006) 1597-1604.

[73] J. Dervaux, Morphogénèse et élasticité en géométrie mince, Ph.D. thesis, Université Paris Diderot (2010).

[74] D. Ruiter, T. Bogenrieder, D. Elder, M. Herlyna, Melanomastroma interactions: structural and functional aspects, Lancet Oncol. 3 (2002) 35-43.

[75] K. Pham, H. B. Frieboes, V. Cristini, J. Lowengrub, Predictions of tumor morphological stability and evaluation against experimental observations, J. Roy. Soc. Interface 8 (2011) 16-29.

[76] A. F. Jerant, J. T. Johnson, C. D. Sheridan, T. J. Caffrey, Early detection and treatment of skin cancer, Am. Fam. Physician 62 (2000) 357-368.

[77] R. Tung, A. Vidimos, Melanoma.

URL http://www.clevelandclinicmeded.com/medicalpubs/diseasemanagement/dermatology/ cutaneous-malignant-melanoma/

[78] G. Argenziano, et al., Slow-growing melanoma: a dermoscopy follow-up study, B. J. Dermatol. 162 (2010) $267-$ 273. 
- The early growth of melanoma is modeled using mixture theory

- Diffusion of nutrients though the epidermis is taken into account

- The effects of endocrine/paracrine regulation mechanisms are investigated

- A linear stability analysis is performed with analytical and numerical techniques

- Predictions on tumor morphology are successfully compared with clinical data 